$$
=
$$

NASA Technical Memorandum 103746

\section{$N-34$ \\ 1668}

940

\title{
Cascade Flutter Analysis With Transient Response Aerodynamics
}

Milind A. Bakhle and Aparajit J. Mahajan

University of Toledo

Toledo, Ohio

Theo G. Keith, Jr.

Ohio Aerospace Institute

Brook Park, Ohio

and University of Toledo

Toledo, Ohio

George L. Stefko

Lewis Research Center.

Cleveland, Ohio

February 1991 


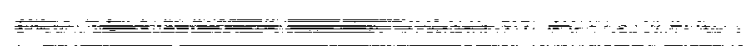

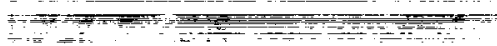
$=-1$
;

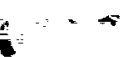




\title{
Cascade Flutter Analysis \\ With Transient Response Aerodynamics
}

\author{
Milind A. Bakhle and Aparajit J. Mahajan \\ University of Toledo \\ Department of Mechanical Engineering \\ Toledo, Ohio 43606 \\ Theo G. Keith, Jr. \\ Ohio Aerospace Institute \\ Brook Park, Ohio 44142 \\ and University of Toledo \\ Department of Mechanical Engineering \\ Toledo, Ohio 43606 \\ George L. Stefko \\ National Aeronautics and Space Administration \\ Lewis Research Center \\ Cleveland, Ohio 44135
}

\begin{abstract}
Two methods for calculating linear frequency domain unsteady aerodynamic coefficients from a time-marching full-potential cascade solver are developed and verified. In the first method, the Influence Coefficient method, solutions to elemental problems are superposed to obtain the solutions for a cascade in which all blades are vibrating with a constant interblade phase angle. The elemental problem consists of a single blade in the cascade oscillating while the other blades remain stationary. In the second method, the Pulse Response method, the response to the transient motion of a blade is used to calculate influence coefficients. This is done by calculating the Fourier transforms of the blade motion and the response. Both methods are validated by comparison with the Harmonic Oscillation method, in which all the airfoils are oscillated, and are found to give accurate results. The aerodynamic coefficients obtained from these methods are used for frequency domain flutter calculations involving a typical section blade structural model. Flutter calculations are performed for two examples over a range of subsonic Mach numbers using both flat plates and actual airfoils.
\end{abstract}




\section{NOMENCLATURE}

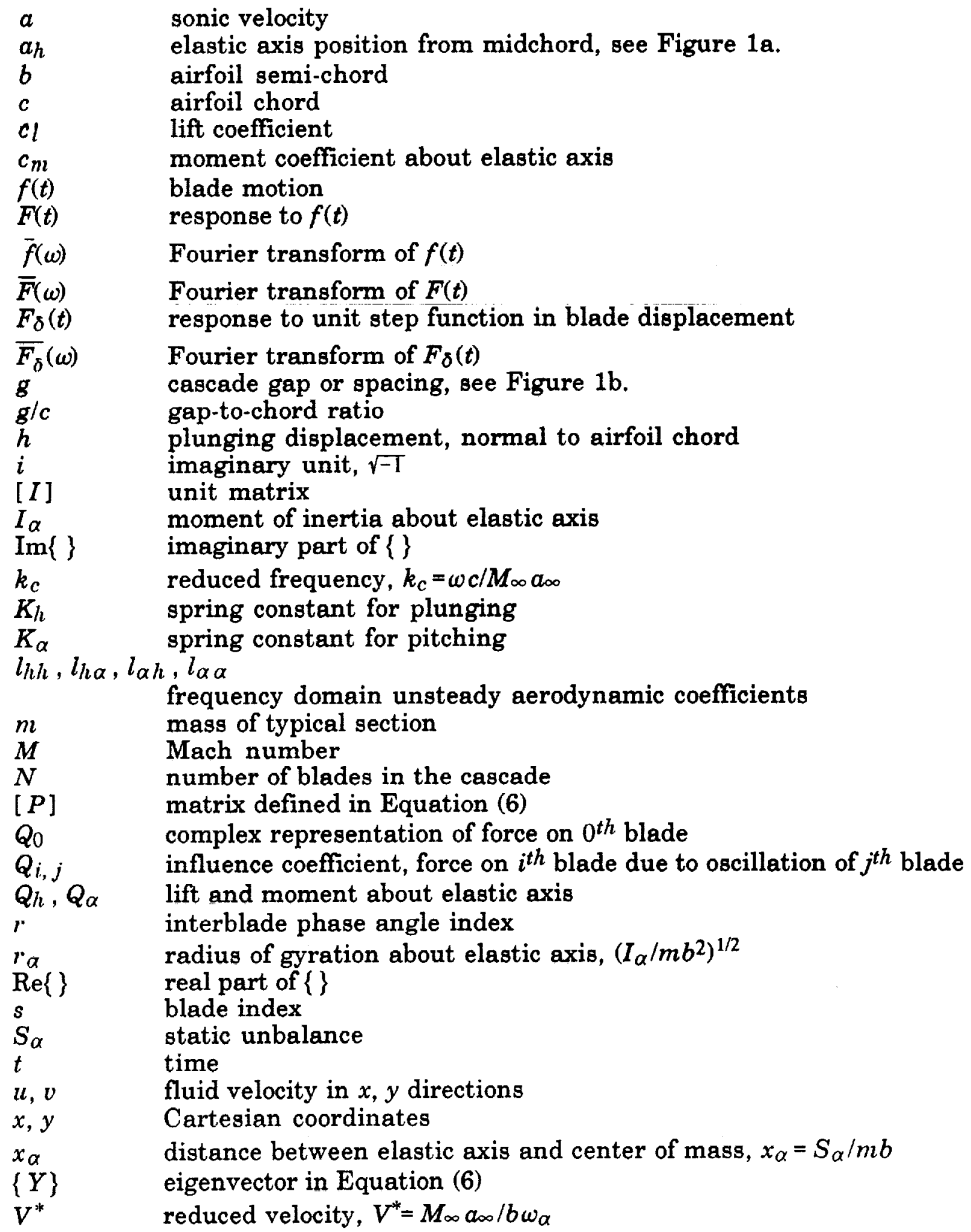


pitching displacement about elastic axis

$\gamma$

$\delta(t)$

ratio of specific heats

$\theta$

unit step function

$\lambda$

stagger angle

$\mu$

$\bar{\mu}$

$\bar{\mu}$

$\bar{v}$

eigenvalue in Equation (6)

mass ratio, $\mu=m / \pi \rho_{\infty} b^{2}$

real part of the eigenvalue (damping ratio) in Equation (7)

imaginary part of the eigenvalue (damped frequency) in Equation (7)

transformed spatial coordinates

$\rho$

fluid (air) density

$\sigma$

$\tau$

interblade phase angle

nondimensional time, $\tau=a_{\infty} t / c$

$\phi$

velocity potential

$\omega$

oscillation frequency

$\omega_{h}$

$\omega_{\alpha}$

( )

()$_{\infty}$

()$_{f}$

()$_{0}$

( )r

()$_{s}$

uncoupled natural frequency for bending (plunging), $\omega_{h}=\left(K_{h} / m\right)^{1 / 2}$ uncoupled natural frequency for torsion (pitching), $\omega_{\alpha}=\left(K_{\alpha} / I_{\alpha}\right)^{1 / 2}$

$d() / d t$

( ) at far upstream conditions

( ) at flutter amplitude of harmonic variable ( )

( ) in the $r^{\text {th }}$ interblade phase angle mode

( ) for the $s^{\text {th }}$ blade 


\section{INTRODUCTION}

Accurate prediction of flutter in turbomachinery components has been a primary research goal of aeroelasticians. The development of advanced turboprop (propfan) engines has led to a renewed interest in the study of flutter in bladed disks. Propfans, which have been designed to operate at high subsonic cruise speeds, have encountered flutter in the early part of their development. ${ }^{1}$

Two fundamental approaches have been used in flutter calculations frequency domain analysis and time domain analysis. The frequency domain method is the conventional approach in turbomachinery aeroelasticity. Recently, the time domain method, which has found wide application in aircraft or fixedwing aeroelasticity, has also been applied to bladed disk or cascade flutter calculations. 2,3 The frequency domain approach has been generally preferred over the time domain approach because the frequency domain analysis requires only unsteady aerodynamic forces corresponding to harmonic blade motion. The time domain method, on the other hand, requires aerodynamic forces corresponding to arbitrary blade motions. The aerodynamic forces corresponding to harmonic blade motions are considerably easier to determine than those corresponding to arbitrary blade motions because the explicit time-dependence can be eliminated from the governing equations, thus reducing the number of independent variables. Further, in a linear flutter analysis, in which only the onset of flutter is to be predicted, blade motions can be assumed to be infinitesimally small. This allows considerable simplification in the aerodynamic analysis since it allows perturbation techniques to be used. For linear flutter analysis, the frequency domain and time domain methods yield the same results. ${ }^{2}$

The unsteady aerodynamic analyses that have been used in flutter calculations have, for the most part, been restricted to inviscid flow theories. Almost all the aerodynamic analyses used in flutter prediction have been based on linearized potential theories. The earliest analyse 4,5 assumed that the unsteady component of the flow was a small harmonic perturbation about a uniform, steady mean flow; this is equivalent to neglecting the blade thickness and camber. Over the past decade, unsteady linearized analyses have been developed ${ }^{6-8}$ that include the effects of non-uniform mean flow due to blade shape. These analyses split the flowfield into steady and unsteady components. The assumption that the unsteady flow component is a small harmonic perturbation about the steady flow allows the elimination of the time variable from the governing equations. The unsteady component of the flow is described by a linear equation with coefficients that depend on the mean steady flowfield.

Recently, unsteady aerodynamic analyses based on the time domain solution of the full-potential and Euler equations have been developed. 9,10 These methods can calculate the unsteady flowfield in a cascade for blade motions that are arbitrary functions of time. The blade motions can be prescribed or determined from a structural model. Accordingly, these flow solvers have been coupled with structural models for time domain flutter analysis.2,11 Also, by specifying the 
blade motions to be simple harmonic (in time), it is possible ${ }^{12}$ to calculate the aerodynamic forces required in a frequency domain flutter analysis. In this case, although the blade motion is prescribed to be harmonic, no assumption is made regarding the linearity of the aerodynamic response (lift and moment). By selecting an amplitude that is sufficiently small to ensure a linear response, it is possible to obtain the same information that was obtained by linearizing the original unsteady equations (by assuming infinitesimal harmonic motions). But, this is not a computationally efficient method for obtaining this type of aerodynamic data for the following reasons. Firstly, the additional independent variable, namely time, adds to the computational cost. Secondly, multiple interblade passages must be included in the calculation to account for the phase lag between the motion of adjacent blades and this increases computational effort considerably. In the present work, alternate methods are developed to calculate the frequency domain unsteady aerodynamic coefficients in a more efficient manner.

An approach has been developed in the present study that allows the time domain full-potential flow solver ${ }^{9}$ to be used efficiently to determine aerodynamic data corresponding to small amplitude harmonic blade motions for use in frequency domain flutter calculations. The conventional approach to calculating harmonic aerodynamic data from a time domain analysis is to specify the blade motions to be simple harmonic and to decompose the resulting time-dependent response into Fourier components. This method, in which all the blades are oscillated with a specified frequency and and interblade phase angle, is referred to as the Harmonic Oscillation method. The Harmonic Oscillation method does not exploit the linearity of the unsteady flow problem for small amplitudes of motion. In the present work, the principle of superposition is used in the Influence Coefficient method to determine the aerodynamic forces for different values of interblade phase angle by summing the solutions to elemental problems. The elemental problem consists of a cascade with one blade oscillating in harmonic motion while the other blades remain stationary. Next, the Pulse Response method is combined with the Influence Coefficient method to determine the aerodynamic coefficients for harmonic blade motions at different values of frequency and different values of interblade phase angle. This is done by giving one of the blades in the cascade a transient motion. The resulting transient forces on all the blades are Fourier transformed and combined to determine the aerodynamic forces at a given frequency and interblade phase angle. These methods are validated by comparison with the Harmonic Oscillation method. Finally, the aerodynamic data thus obtained is used for flutter calculations. Flutter calculations are done with a typical section structural model (lumped parameter representation) for each blade. Two examples are considered, one with a single-degree-of-freedom typical section and the other with a two-degrees-offreedom typical section. The structural parameters and airfoil shape used in the second example are representative of the SR5 propfan. ${ }^{13}$ 


\section{ANALYSIS}

Flutter analysis of a fluid-structure system requires an aeroelastic representation of the system. Accordingly, the structural model, the aerodynamic model and the aeroelastic stability analysis are described in this section.

\section{Structural model}

Each blade of the rotor (bladed disk) is represented by a typical section mode $1^{14}$. The dynamic behavior of the blade is represented by a rigid twodimensional airfoil section taken from the three-quarter span location of the blade. The typical section is allowed two degrees of freedom - plunging and pitching as shown in Figure 1a. The plunging motion is in a direction perpendicular to the airfoil chord and models the bending of the blade; the pitching motion about the elastic axis simulates the torsion of the blade. The equations of motion for a single blade are:

$$
\begin{aligned}
& m \ddot{h}+S_{\alpha} \ddot{\alpha}+K_{h} h=Q_{h} \\
& S_{\alpha} \ddot{h}+I_{\alpha} \ddot{\alpha}+K_{\alpha} \alpha=Q_{\alpha}
\end{aligned}
$$

where $h$ is the plunging (bending) displacement, $\alpha$ is the pitching (torsion) displacement, $m$ is the airfoil mass, $I_{\alpha}$ is the moment of inertia, $S_{\alpha}$ is the static unbalance, $K_{h}$ and $K_{\alpha}$ are the spring constants for plunging and pitching, respectively; $Q_{h}$ and $Q_{\alpha}$ are the aerodynamic loads (lift and moment, respectively) and the dots over the displacements indicate differentiation with respect to time.

\section{Aerodynamic model}

The aerodynamic model is based on the unsteady, two-dimensional, fullpotential equation. The governing equation for irrotational, isentropic flow written in conservative form is:

$$
\frac{\partial \rho}{\partial t}+\frac{\partial(\rho u)}{\partial x}+\frac{\partial(\rho v)}{\partial y}=0
$$

where

$$
u=\frac{\partial \phi}{\partial x}, v=\frac{\partial \phi}{\partial y} \text { and } \frac{\rho}{\rho_{\infty}}=\left\{1+\frac{(\gamma-1)}{2}\left[M_{\infty}^{2}-\left(2 \frac{\partial \phi}{\partial t}+\left(\frac{\partial \phi}{\partial x}\right)^{2}+\left(\frac{\partial \phi}{\partial y}\right)^{2}\right) / a_{\infty}^{2}\right]\right\}^{1 /(\gamma-1)}
$$


In these equations, $\phi$ is the velocity potential; $\rho_{\infty}, a_{\infty}$ and $M_{\infty}$ are the density, sonic velocity and Mach number, respectively, all evaluated at far upstream conditions.

The governing equation is transformed to the computational plane where it is discretized and solved using a finite-volume scheme. The method of solution ${ }^{9,15}$ is a time-marching scheme that uses approximate factorization at each time level with quasi-Newton iterations to maintain time accuracy. The transformation from physical coordinates $(x, y, t)$ to computational coordinates $(\xi, \eta, \tau)$ is defined at discrete grid points. An H-type grid, shown schematically in Figure 1b, is used. It consists of $\xi=$ constant lines that are parallel to the $\mathrm{y}$-axis and $\eta=$ constant lines that are obtained by interpolating between the upper and lower boundaries of each interblade passage. For unsteady calculations, the airfoil positions change with time and a new grid is generated at each time level by interpolating between the passage boundaries determined from the instantaneous positions of the airfoils.

The governing equation is solved as an initial-boundary value problem. For steady calculations, a uniform flowfield is used as the initial condition; for unsteady calculations, the steady flowfield is used as the initial condition. The boundary conditions are described below.

\section{Airfoil surface:}

The airfoil surfaces are treated as impermeable, i.e., the normal velocity of the fluid relative to the airfoil surface is zero. Flow tangency is imposed at the instantaneous position of the airfoil at each time step. In order to affect this, a new grid which conforms to the airfoil surfaces is generated at each time step. Blade motions are thus fully accounted for using moving grids.

\section{Wake:}

The vorticity that is shed from the trailing edge of the airfoil is assumed to remain confined within an infinitely thin region of the flowfield denoted as a wake sheet. Wakes are free material surfaces with no pressure difference across them. In the present analysis, wake positions are prescribed in advance; continuity of pressure and normal velocity is then enforced across the wake. This procedure is followed to avoid difficulties related to the tracking of the wake location at each time step. The wakes are prescribed to be straight lines extending from the trailing edge of each airfoil to the exit boundary. The slope of these lines is selected to be the mean of the slopes of the upper and lower surfaces of the airfoil at the trailing edge.

\section{Inlet/Exit boundaries:}

The inlet and exit boundaries are artificial boundaries that have been introduced because the numerical computations cannot be extended to infinite distances in the upstream and downstream directions and must be restricted to a small, finite region in space. Since these are non-physical boundaries, the boundary conditions that are used must allow acoustic waves to pass through without reflecting them back into the computational region. Even though it would be convenient to prescribe the far upstream/downstream flow velocity or Mach number at these boundaries, this is not done because it leads to non-physical wave 
reflections. In the present analysis, characteristic boundary conditions ${ }^{16}$ are used at the inlet and exit boundaries. At the inlet/exit boundary, the Riemann invariant corresponding to the incoming characteristic (positive characteristic with respect to the inward normal) is prescribed based on the far upstream/downstream conditions. This allows planar acoustic waves, which are at normal incidence to the boundary, to pass through without reflection. However, the acoustic waves are generally not planar; also, they are generally not at normal incidence to the boundary. Therefore, the use of one-dimensional Riemann-invariant boundary conditions results in some wave reflections back into the calculation domain.

Periodic boundaries:

Periodic conditions are imposed on grid lines extending from the leading edge of the airfoil to the inlet boundary; see Figure 1b. The periodic conditions are used to simulate the fundamental periodicity present on the bladed disk in the circumferential direction.

Additional details concerning the aerodynamic model and the boundary conditions can be found in References 9 and 15.

\section{Aeroelastic Stability}

For a tuned cascade, in which all the blades are identical, the aeroelastic modes consist of each of the $N$ individual blades vibrating with equal amplitudes with a fixed interblade phase angle between adjacent blades 17 . For the $s^{\text {th }}$ blade vibrating in the $r^{t h}$ interblade phase angle mode, this can be written as:

$$
\left|\begin{array}{c}
h_{s} / b \\
\alpha_{s}
\end{array}\right|=\left\{\begin{array}{c}
h_{o} / b \\
\alpha_{0}
\end{array} \mid e^{i\left(\omega t+\sigma_{r} s\right)} \quad s=0,1,2, \ldots, N-1\right.
$$

The phase angle between adjacent blades is given as

$$
\sigma_{r}=2 \pi r / N \quad r=0,1,2, \ldots, N-1
$$

The corresponding aerodynamic forces can be written as linear functions of the displacements using the complex-valued frequency domain unsteady aerodynamic coefficients $l_{h h}, l_{h \alpha}, l_{\alpha h}$ and $l_{\alpha \alpha}$ :

$\left.\mid \begin{array}{c}Q_{h s} / m b \\ Q_{\alpha s} / m b^{2}\end{array}\right\}=\frac{\omega^{2}}{\mu}\left\{\begin{array}{l}l_{h h r} h_{o} / b+l_{h \alpha r} \alpha_{o} \\ l_{\alpha h r} h_{o} / b+l_{\alpha \alpha r} \alpha_{o}\end{array}\right\} e^{i\left(\omega t+\sigma_{r} s\right)}$

where $\mu=m / \pi \rho_{\infty} b^{2}$ is the mass ratio of the blade. The eigenvalue problem for the tuned system can be written 18 as: 


$$
[[P]-\lambda[I]]\{Y\}=\{0\}
$$

where

$$
[P]=\left[\begin{array}{cc}
\frac{\mu+l_{h h r}}{\mu\left(\omega_{h} / \omega_{\alpha}\right)^{2}} & \frac{\mu x_{\alpha}+l_{h \alpha r}}{\mu\left(\omega_{h} / \omega_{\alpha}\right)^{2}} \\
\frac{\mu x_{\alpha}+l_{\alpha h r}}{\mu r_{\alpha}^{2}} & \frac{\mu r_{\alpha}^{2}+l_{\alpha \alpha r}}{\mu r_{\alpha}^{2}}
\end{array}\right]
$$

In the above, $x_{\alpha}=S_{\alpha} / m b$ is the distance between the elastic axis and center of mass and $r_{\alpha}=\left(I_{\alpha} / m b^{2}\right)^{1 / 2}$ is the radius of gyration about the elastic axis, both in semi-chord units; $\omega_{h}=\left(K_{h} / m\right)^{1 / 2}$ and $\omega_{\alpha}=\left(K_{\alpha} / I_{\alpha}\right)^{1 / 2}$ are the uncoupled natural frequencies for bending and torsion, respectively; note, the subscript 's' which identifies the blade has been dropped.

For each interblade phase angle mode, the solution of the above eigenvalue problem results in two complex eigenvalues of the form

$$
i \frac{\omega}{\omega_{\alpha}}=\frac{i}{\sqrt{\lambda}}=\bar{\mu}+i \bar{v}
$$

The real part of the eigenvalue $(\bar{\mu})$ represents the damping ratio and the imaginary part $(\bar{v})$ represents the damped frequency; flutter occurs if $\bar{\mu} \geq 0$ for any of the eigenvalues. The stability of each phase angle mode is examined separately. The interblade phase angle is fixed at one of the values given by Equation (5) and the $2 \times 2$ eigenvalue problem is solved.

However, before the eigenvalue problem can be solved, the aerodynamic coefficients must be calculated. For fixed cascade geometric parameters, the aerodynamic coefficients are functions of inlet Mach number $\left(M_{\infty}\right)$, reduced frequency of blade vibration $\left(k_{c}\right)$ and interblade phase angle $\left(\sigma_{r}\right)$. The following procedure has been adopted for flutter calculations. A value of inlet Mach number is selected. A value of reduced frequency is assumed and the aerodynamic coefficients $l_{h h}, l_{h \alpha}, l_{\alpha h}$ and $l_{\alpha \alpha}$ are calculated for all values of phase angle in Equation (5). The eigenvalue problem is solved for each value of interblade phase angle. The reduced frequency is varied until the real part of one of the eigenvalues $(\bar{\mu})$ becomes zero while the real part of the other eigenvalue is negative. The assumed flutter reduced frequency $\left(k_{c f}\right)$ and the calculated flutter frequency $\left(\bar{v}_{f}\right)$ are both based on $\omega_{f}$. Thus, these two can be combined to eliminate $\omega_{f}$ and the flutter reduced velocity $\left(V_{f}^{*}\right)$ is obtained, viz., $V_{f}^{*}=2 \bar{v}_{f} / k_{c f}$. The critical phase angle is identified as the one which results in the lowest flutter velocity. The stability of a single-degree-of-freedom pitching system can be inferred entirely 
from the imaginary part of the moment coefficient, $\operatorname{Im}\left\{l_{\alpha \alpha}\right\}$; flutter occurs if $\operatorname{Im}\left\{l_{\alpha a}\right\} \geq 0$.

\section{Aerodynamic Coefficients}

The calculation of the frequency domain unsteady aerodynamic coefficients $\left(l_{h h}, l_{h \alpha}, l_{\alpha h}\right.$ and $\left.l_{\alpha \alpha}\right)$ is described next. For a given cascade geometry and inlet Mach number, these coefficients are required for plunging and pitching motions of specified frequency and specified interblade phase angle (restricted by Equation (5)). The full-potential solver is based on a time-marching algorithm and three different methods of calculating the aerodynamic coefficients are described.

\section{Harmonic Oscillation Method}

In the Harmonic Oscillation (HO) method 12 , all the $N$ airfoils are oscillated with the specified frequency and specified interblade phase angle. The motion of the $s^{\text {th }}$ airfoil in the cascade is prescribed to be of the form:

$$
\begin{aligned}
& h=h_{o} \sin \left(\omega t+s \sigma_{r}\right)=h_{o} \sin \left(k_{c} M_{\infty} \tau+s \sigma_{r}\right) \quad \text { for plunging } \\
& \alpha=\alpha_{o} \sin \left(\omega t+s \sigma_{r}\right)=\alpha_{o} \sin \left(k_{c} M_{\infty} \tau+s \sigma_{r}\right) \quad \text { for pitching }
\end{aligned}
$$

Calculations are started from a steady flowfield and continued until starting, nonperiodic, transients have decayed and the flowfield has become periodic in time. The lift and moment acting on the reference airfoil $(s=0)$ are calculated at every time step and later decomposed into Fourier components. If the amplitude of oscillations is sufficiently small, it can be shown by a perturbation analysis that the flowfield will have the same harmonic time dependence as the motion ${ }^{7}$. Therefore, the lift and moment coefficients $\left(c_{l}\right.$ and $c_{m}$ ) on the zeroth blade can be represented in terms of a complex quantity $Q_{0}$ as $\left[\operatorname{Im}\left\{Q_{0}\right\} \cos (\omega t)+\right.$ $\left.\operatorname{Re}\left\{Q_{0}\right\} \sin (\omega t)\right]$. The frequency domain unsteady aerodynamic coefficients $\left(l_{h h}\right.$, $l_{h \alpha}, l_{\alpha h}$ and $\left.l_{\alpha \alpha}\right)$ are then obtained by dividing $Q_{0}$ by $k_{c}{ }^{2}$, the amplitude of motion and other constants. The harmonic oscillation method requires that calculations for each of the $N$ values of interblade phase angle be done separately. It is possible to reduce this computational effort by using the Influence Coefficient method described next.

\section{Influence Coefficient Method}

The Influence Coefficient (IC) method is based on the principle of linear superposition. Briefly, the solution to a problem is obtained by superposing the solutions to the individual elemental problems that comprise the original problem. Since the method is based on the principle of linear superposition, it is valid only for linear problems. It can be shown that the unsteady part of the present problem is linear (governed by a linear differential equation) for 
sufficiently small amplitude of oscillation. ${ }^{7}$ It should be emphasized that only the unsteady part of the problem is linear; the steady flowfield is described by a nonlinear equation.

Since the quantities of significance are the lift and moment coefficients, the following discussion will deal only with a general integrated force quantity. However, the results obtained can be extended by analogy to pressure distributions or the distribution of other flow variables. Also, complex notation is used for convenience; in this notation it is implied that only the imaginary part of the complex quantity is to be considered. For example, when the motion is specified to be of the form $h_{o} e^{i \omega t}$, it is understood that the motion is actually $h_{o} \sin (\omega t)$.

The problem to be solved consists of a cascade of $N$ blades in which each blade oscillates with a motion of the form $\sin \left(\omega t+s \sigma_{r}\right)$, where $s$ is the blade index that varies from 0 to $N-1$, and $\sigma_{r}$ is the interblade phase angle given by Equation (5). This problem is divided into $N$ elemental problems. The $k^{\text {th }}$ elemental problem consists of the same cascade of $N$ blades in which the $k^{\text {th }}$ blade oscillates with a motion of the form $\sin (\omega t)$ while all other blades remain stationary. The original problem and all the elemental problems have solutions that are harmonic functions of time.

For the problem in which all blades oscillate with a motion of the form $e^{i\left(\omega t+s \sigma_{r}\right)}$, the forces $\left(c_{l}\right.$ and $\left.c_{m}\right)$ on the $0^{t h}$ blade can be represented as $Q_{0} e^{i \omega t} ; Q_{0}$ is complex valued to allow the force to lead or lag the motion. The forces on the $0^{\text {th }}$ blade in the $k^{t h}$ elemental problem can be represented as $Q_{0, k} e^{i \omega t} ; Q_{0, k}$ is sometimes referred to as an influence coefficient. Thus, using superposition, the following relation can be obtained.

$$
Q_{0} e^{i \omega t}=\sum_{k=0}^{N-1} Q_{0, k} e^{i \omega t} e^{i k \sigma_{r}}
$$

Now, due to the periodicity of the cascade, only the relative positions of the oscillating blade and the reference (zeroth) blade are important. That is, the forces generated on the $0^{t h}$ blade due to the oscillation of the $k^{t h}$ blade are equal to the forces on the $1^{s t}$ blade due to the oscillation of the $k+1^{\text {th }}$ blade, and so on. Thus,

$$
Q_{0, k}=Q_{-k, 0}=Q_{N-k, 0}
$$

where the periodicity of the cascade of $N$ blades has been invoked again in the last step. Thus, the solution to the problem in terms of the influence coefficients can be written as:

$$
Q_{0} e^{i \omega t}=\sum_{k=1}^{N} Q_{N-k, 0} e^{i \omega t} e^{i k \sigma_{r}}
$$


Replacing the influence coefficients $Q_{0, k}$ by the coefficients $Q_{N-k, 0}$ means that all the required influence coefficients can be determined simultaneously rather than separately. Thus, instead of oscillating the $k^{\text {th }}$ blade, calculating the pressure history on the zeroth blade and then repeating for all values of $k$ between 0 and $N-1$, it is possible to oscillate the zeroth blade and calculate the pressures on all the blades simultaneously. This means that the computational effort required for the calculation of all the influence coefficients can be reduced by a factor of $N$ over the Harmonic Oscillation method.

It should be noted that the elemental problem used in the present work is different from the one used more frequently, in which a single blade in an infinite cascade is oscillated 19,20 . In the present work, periodic boundary conditions are used to simulate an infinite cascade using $N$ blades in the calculations. Thus, when a single blade in the $N$-blade cascade is oscillated, it corresponds to every $N^{t h}$ blade in the infinite cascade being oscillated. The expressions presented in this section are exact for the discrete values of interblade phase angle given by Equation (5) and approximate for all other values. Although the summation in Equation (8) only extends over a finite number of terms, it does not represent a truncation of an infinite sum as long as the value of interblade phase angle is restricted by Equation (5). Calculations performed without using periodic boundary conditions simulate a finite section of an infinite cascade surrounding the blade that is being oscillated. This introduces an approximation into such calculations for all values of interblade phase angle; however, this error decreases rapidly as the number of blades used in the calculations is increased.

\section{Pulse Response Method}

For a given motion, plunging or pitching, the Harmonic Oscillation method and the Influence Coefficient method require separate calculations for each oscillation frequency of interest. In order to reduce the computational effort, the Pulse Response (PR) method described in this section can be used; this method has evolved from the indicial approach that is widely used in many different fields.

Researchers have investigated the indicial approach for aerodynamic calculations with isolated airfoils 21 and cascades 22 . The indicial response is the response, lift or moment, to a step change in the given mode of motion. From the indicial response, the response for any arbitrary motion, specifically harmonic motion, can be calculated using Duhamel's superposition integral. Let the timedependence of the blade motion (plunging or pitching) be denoted as $f(t)$ and let the corresponding response (lift or moment) be denoted as $F(t)$. Let $F_{\delta}(t)$ denote the response corresponding to a unit step function, $f(t)=\delta(t)$. The response corresponding to an arbitrary motion $f(t)$ can then be written using Duhamel's superposition integral as

$$
F(t)=\int_{0}^{t} F_{\delta}(t-\zeta) \dot{f}(\zeta) d \zeta
$$


Using the above equation, the response to a harmonic motion, $f(t)=e^{i \omega t}$, can be determined. Since only the periodic response is of interest, the limit of the above integral as $t \rightarrow \infty$ is considered. Using a change of variable and extending the lower limit to $-\infty$, the following relation can be obtained

$$
F(t)=i \omega \overline{F_{\delta}}(\omega) e^{i \omega t}
$$

where $\overline{F_{\delta}}(\omega)$ is the Fourier transform of $F_{\delta}(t)$ given by

$$
\overline{F_{\delta}}(\omega)=\int_{-\infty}^{+\infty} F_{\delta}(t) e^{-i \omega t} d t
$$

The indicial response method has the following drawback. The step change in the displacement results in infinite velocity at the time at which the step change occurs. The indicial response contains a large spike at this time. Therefore, very small time steps must be used to ensure that the results are accurate during this period of rapid transients and the accurate evaluation of the Fourier integral over this time interval is difficult. In addition, the treatment of this infinite velocity by finite differences leads to non-physical transients that can cause errors in the final results. To avoid these difficulties, researchers ${ }^{23}$ have replaced the step function by a smooth version which does not result in infinite velocities and spikes. Polynomial functions of time have been used in place of the standard step function to obtain the necessary smoothness at the beginning and end of the step. This allows time steps of normal size to be used in the calculations.

For an arbitrary motion $f(t)$ and the corresponding response $F(t)$, the Fourier transform of Equation (11) gives ${ }^{24}$ :

$$
i \omega \overline{F_{\delta}}(\omega)=\bar{F}(\omega) / \bar{f}(\omega)
$$

where $\bar{f}(\omega)$ and $\bar{F}(\omega)$ are the Fourier transforms of $f(t)$ and $F(t)$, respectively. It should be noted from Equation (12) that $i \omega \overline{F_{\delta}}(\omega)$ gives the response to a harmonic motion. Since only the response to a harmonic motion is to be obtained, any arbitrary motion and the corresponding response can be used to obtain $i \omega \overline{F_{\delta}}(\omega)$ from Equation (14).

Thus, the time variation of both the motion and the response are required to calculate the response to harmonic motions. To reduce the time required for the transients to decay, the smooth step is often replaced by a pulse 25 . In the pulse motion, the blade returns to its original position after the duration of the pulse. This is in contrast to the step motion in which the blade position is different before and after the step. The pulse motion thus allows the flowfield to return to its steady undisturbed state after the transients created by the pulse have decayed. The unsteady calculations therefore need to be carried out only long enough to 
ensure that the solution has reached its final state (the same as the initial state) within some specified tolerance.

Several smooth pulse shapes have been investigated by researchers. A comparison $^{3}$ of results from three different pulse shapes shows that although the shape and size of the pulse determines the transient response, the ratio of the Fourier transforms of the response and the motion remains unchanged. Thus, the shape of the pulse is not of particular importance although care must be taken to ensure that the transform of the pulse motion does not become zero in the frequency range of interest. In addition, the duration of the pulse must be selected according to the range of frequencies of interest. Thus, the harmonic time period of interest must be smaller than the duration of the pulse for the results to be meaningful. This places a lower limit on the values of frequency for which calculations can be made using a pulse of given duration. The upper limit on the frequency is determined by the size of the time step; the upper limit is generally not relevant because the time step is normally quite small for reasons of numerical stability and accuracy.

In the present calculations, the pulse function is selected as:

$$
\begin{array}{ll}
f(t)=4\left(\frac{t}{t_{\max }}\right)^{2} \exp \left(2-\frac{1}{1-t / t_{\max }}\right) & \text { for } 0 \leq t<t_{\max } \\
f(t)=0 & \text { for } t \geq \operatorname{tmax}
\end{array}
$$

where $t_{\max }$ is the duration of the pulse. The above choice makes $f(t)$ and $\dot{f}(t)$ vanish at $t=0$ and $t=t_{\max }$; in addition, higher derivatives also go to zero at $t=t_{\max }$. This ensures that there is a smooth transition to and from the undisturbed blade position.

The Pulse Response method is used in conjunction with the Influence Coefficient method as follows. One blade in the cascade is given a transient motion of the form $h(t)=h_{o} f(t)$ or $\alpha(t)=\alpha_{o} f(t)$. The calculations start with the steady solution and unsteady response to the pulse in either motion, plunging or pitching, is calculated until the transient flowfield reaches the steady flowfield within a specified tolerance. The motion as well as the responses on all the blades are recorded and Fourier transforms of these are calculated numerically for the frequency of interest. Using these transforms, the influence coefficients $\left(Q_{k, 0}\right)$ are calculated from Equations (14); it is to be noted that the harmonic response, $i \omega \overline{F_{\delta}}(\omega)$, obtained from Equation (14) for this case, is simply the influence coefficient, $Q_{k, 0}$. Equation (10) is then used to calculate the frequency domain unsteady aerodynamic coefficients $\left(l_{h h}, l_{h \alpha}, l_{\alpha h}\right.$ and $\left.l_{\alpha \alpha}\right)$ for the interblade phase angle of interest. In this way, the coefficients can be determined for various values of reduced frequency by recalculating the Fourier transforms for the frequency of interest using the same time histories. 


\section{RESULTS AND DISCUSSION}

The results are presented in two parts. The results in the initial part serve to validate the Influence Coefficient method and the Pulse Response method by comparison with the Harmonic Oscillation method. In the remaining part of this section, results of flutter calculations for two examples are presented.

\section{Calculation of Aerodynamic Coefficients}

Figure 2 shows the unsteady lift coefficient due to plunging motion in a cascade comprised of eight flat plates. The cascade is unstaggered and has a unity gap-to-chord ratio. The Mach number of the flow at the inlet is $M_{\infty}=0.5$ and the reduced frequency of oscillation is $k_{c}=1.0$. A $41 \times 21$ grid is used in the calculations with 41 points in the streamwise direction and 21 points in the direction of the stagger line in each of the 8 interblade passages; 21 points are located on each (upper and lower) surface of each airfoil; all grid points are uniformly distributed. 150 time steps/cycle of oscillation are used which corresponds to a nondimensional time step of 0.084 and the calculations are continued for three cycles of blade oscillation to allow non-periodic starting transients to decay. The amplitude of oscillation used is $h_{0} / c=0.002$ which is found to be small enough to yield a response which is linearly dependent on the amplitude of motion.

The results obtained from the IC method and the HO method are represented as open symbols; the results from classical linear theory 4 are shown as dashed lines and denoted as 'linear [4]'. A comparison of the various results reveals the following. The HO method and the IC method yield virtually identical results. This is simply a verification of the superposition principle and the associated calculations. It implicitly confirms that the unsteady problem is linear for the amplitude of motion used in the calculations. Similar comparisons made with other airfoils and other cascade geometries have demonstrated the same level of agreement seen here. The results of the present calculations also agree quite well with the results from classical linear theory. For flat plate airfoils and small amplitude oscillations, the present calculations are expected to yield the same results as obtained from classical linear theory. It is to be noted that classical linear theory uses a semi-analytical formulation and the solutions show singular behavior near the leading edge. This feature is not completely captured by the relatively coarse $41 \times 21$ grid used in the present calculations. This may account for some of the differences observed between the results. Figure 3 shows the moment coefficient for the plunging motion. Figures 4 and 5 show the lift and moment coefficients due to pitching about midchord; the amplitude of pitching oscillations used in the calculations is $\alpha_{o}=0.2^{\circ}$ and 210 time steps/cycle of oscillation are used. In all the above results, the agreement between the HO method and the IC method is extremely good. The agreement between the present results and those from classical linear theory ranges from fair to good. 
Figure 6 shows the time-variation of the displacement and the resulting lift on all the blades of a 8-blade cascade of flat plates for use in the PR method. The grid and the time step used are the same as those used in the previous calculations with the HO method and the IC method. The motion of blade 5 is shown as a function of time; the other blades in the cascade remain stationary. The maximum displacement during the pulse is $h_{o} / c=0.002$ and the duration of the pulse is $t_{\max }=20 \pi$. The lift on all blades is shown separately. As can be seen, the calculations are continued until all responses have returned to the initial undisturbed values. For the present calculation, the initial and final response levels correspond to zero lift since the airfoils are flat plates. If the calculations are for a configuration which has a non-zero value of steady state lift, then similar variations will be obtained for the unsteady component of the lift (instantaneous lift minus steady lift). The time histories are Fourier transformed and combined according to Equation (14) to obtain the influence coefficients $\left(Q_{N-k, 0}\right)$ which are combined according to Equation $(10)$ to get the harmonic coefficients.

Figure 7 shows the lift due to plunging obtained by applying the PR method and the IC method to the time histories of Figure 6 . The solid lines are results from the PR+IC method and the open symbols represent results from the HO method. Results from classical linear theory are also shown for comparison. The agreement between the PR+IC method and the HO method is very good. This substantiates the validity of the PR method used in combination with the IC method. It also implicitly confirms the linearity of the unsteady problem which permits the use of the Duhamel superposition integral and the influence coefficient approach. The agreement between the present calculations and the results from classical linear theory is also quite good except at values of reduced frequency near the point of acoustic resonance. The comments made earlier regarding the singularity near the leading edge and the limitations of the grid used in the present study can be applied to account for some of the observed discrepancy. Figures 8-10 show the variation of the remaining coefficients for the same cascade and flow condition. As before, the agreement between the PR+IC method and the HO method is very good and the agreement between the present results and those from classical linear theory varies from fair to good.

All the computations described here were performed on a CRAY X-MP computer. The calculations performed using the HO method required about 160 CPU seconds for the case of pitching motion with $k_{c}=1.0$ and $\sigma=45^{\circ}$. Approximately the same amount of time was required for the corresponding calculations using the IC method with $k_{c}=1.0$ which gave results for $\sigma=0^{\circ}, 45^{\circ}, 90^{\circ}, \ldots, 315^{\circ}$. The calculation of the time histories in the PR method required about $230 \mathrm{CPU}$ seconds; the additional time required for calculating Fourier transforms was quite small and was not recorded. 


\section{Calculation of Flutter Boundaries}

Flutter results are presented for two examples. The first example is a singledegree-of-freedom (pitching) system that has been previously considered in References 2 and 12. The cascade has nine blades, a stagger angle of $\theta=45^{\circ}$, a gapto-chord ratio of $g / c=1.0$ and the elastic axis is located at the leading edge $\left(a_{h}=-1.0\right)$. The stability of this system can be inferred entirely from the imaginary part of the moment coefficient, $\operatorname{Im}\left\{l_{\alpha \alpha}\right\}$. Flutter occurs if $\operatorname{Im}\left\{l_{\alpha \alpha}\right\} \geq 0$ in which case the oscillating cascade extracts energy from the fluid stream. Figure 11 shows the moment coefficient (real and imaginary parts) at the different values of interblade phase angle that may arise at flutter (Equation (5)). The results are for an inlet Mach number of $M_{\infty}=0.5$ and a reduced frequency of $k_{c}=0.222$. Calculations have been performed on three different grids with $41 \times 21,81 \times 41$, and $121 \times 61$ points in each interblade passage; the number of grid points on each airfoil surface (upper and lower) is 21,41 and 61 , respectively. The results from classical linear theory are included for comparison.

It can be seen from Figure 11 that a uniform refinement of the grid spacing by a factor of 3 results only in small changes in the value of the moment coefficient. Some discrepancy is also observed between the present results and the results obtained from classical linear theory; this difference is not always reduced by grid refinement. This indicates that some other cause exists for the observed discrepancy. Efforts are currently underway to resolve this difficulty; no explanation is offered at present. It should be noted that observed difference cannot be attributed to the use of the PR method or the IC method since these have been validated by comparison with the HO method.

For the calculations performed using the $41 \times 21$ grid, a reduced frequency of $k_{c}=0.222$ results in neutral stability $\left(\operatorname{Im}\left\{l_{\alpha \alpha}\right\}=0\right)$ of the $\sigma=320^{\circ}$ aeroelastic mode with all other modes being stable $\left(\operatorname{Im}\left\{l_{\alpha \alpha}\right\}<0\right)$; this result was obtained after calculating the coefficient $l_{\alpha} \alpha$ at different values of reduced frequency and interblade phase angle. Thus, the reduced frequency at flutter is $k_{c f}=0.222$ and the phase angle at flutter is $\sigma_{f}=320^{\circ}$. The corresponding results from classical linear theory are $k_{c f}=0.254$ and $\sigma_{f}=320^{\circ}$. Similar calculations have been performed for other values of the inlet Mach number between 0.2 and 0.8 using a $81 \times 41$ grid in each interblade passage. Figure 12 shows the variation of flutter reduced frequency with Mach number. It can be seen that the reduced frequency at flutter increases with the inlet Mach number with a levelling-off at the higher values of Mach number. In addition to the flat plate airfoil, results are also shown for a double-circular-arc airfoil with thickness-to-chord ratio of 0.05 (5\% DCA airfoil). These calculations have also been performed with a $81 \times 41$ grid in each interblade passage. In the present example, the effect of thickness is to increase the flutter reduced frequency at all the values of inlet Mach number. The interblade phase angle at flutter was found to be $\sigma_{f}=320^{\circ}$ for all the results shown. 
The second example for which flutter calculations have been performed consists of a 5-blade cascade in which the typical section has two degrees-offreedom. This example has been previously considered in Reference 2; the geometric and structural parameters in this example are representative of the SR5 propfan ${ }^{13}$. The cascade stagger angle is $\theta=10.7^{\circ}$ and the gap-to-chord ratio is $g / c=1.85$. The airfoil section is from a NACA 16 series with a thickness-to-chord ratio of 0.03 and a design lift coefficient of 0.3 ; this airfoil, which is taken from the three-quarter span location of the SR5 propfan, is hereafter referred to as the SR5 airfoil. The structural model for each blade is a two-degrees-of-freedom typical section with elastic axis at the leading edge $\left(a_{h}=-1.0\right)$. The mass ratio is $\mu=115$, the radius of gyration is $r_{\alpha}=1.076$, the offset between elastic axis and center of mass is $x_{\alpha}=0.964$ and the ratio of uncoupled natural frequencies in bending and torsion is $\omega_{h} / \omega_{\alpha}=0.567$.

The following procedure has been used in the calculations. For each value of interblade phase angle obtained from Equation (5), the reduced frequency is varied until one of the two eigenvalues of Equation (7) displays neutral stability $(\bar{\mu}=0)$ while the other eigenvalue displays stability $(\bar{\mu}<0)$. The reduced frequency thus obtained $\left(k_{c f}\right)$ is shown in Figure 13 for a flat plate airfoil. Calculations have been done using three different grids with $41 \times 38,61 \times 57$, and $81 \times 75$ points in each of the five interblade passages; the number of grid points on each airfoil surface (upper and lower) is 21,31 and 41 , respectively. Results from classical linear theory are included for comparion. It can be seen that reducing the grid spacing by a factor of 2 does not result in much change in the reduced frequency at flutter. Figure 14 shows the flutter reduced velocity for the same case. It can be seen that the lowest flutter reduced velocity occurs at $\sigma=288^{\circ}$ and this is identified as the critical phase angle. The corresponding flutter reduced velocity is $V_{f}^{*}=7.82$ for the $41 \times 38$ grid, $V_{f}^{*}=7.71$ for the $61 \times 57$ grid and $V_{f}^{*}=7.63$ for the $81 \times 75$ grid; the result from classical linear theory is $V_{f}^{*}=7.01$.

Similar calculations have been performed for values of inlet Mach number between 0.2 and 0.8 ; a $61 \times 57$ grid is used. Calculations are done for a flat plate airfoil and the SR5 airfoil. The results are presented in Figures 15 and 16 as variations of flutter reduced velocity $\left(V_{f}^{*}\right)$ and flutter frequency ratio $\left(\omega_{f} / \omega_{\alpha}\right)$ with Mach number. Figure 15 shows that the flutter reduced velocity decreases with increasing Mach number for both the flat plate airfoil and the SR5 airfoil; the variation is seen to be almost linear. Figure 16 shows that the flutter frequency ratio also decreases with increasing Mach number. It is noted that there is only a marginal difference between the results for the flat plate and the SR5 airfoil. However, it may be recalled that the SR5 airfoil has a small camber angle, a small thickness-to-chord ratio of 0.03 and the cascade gap-to-chord ratio is large $(g / c=1.85)$. Therefore, it may be concluded that there is not much steady flow deflection for this case and consequently the flutter results do not show much difference between the flat plate airfoil and the SR5 airfoil. 


\section{CONCLUDING REMARKS}

Two methods of calculating (linear) frequency domain unsteady aerodynamic coefficients from a time-marching full-potential solver have been developed and verified. The first method, the Influence Coefficient method, allows coefficients for different interblade phase angles to be calculated simultaneously. The second method, the Pulse Response method, allows coefficients for several oscillation frequencies to be calculated from the same transient response. When these two methods are combined, the aerodynamic coefficients for several combinations of phase angles and frequencies can be calculated at approximately the computational cost required for the calculation of a single phase angle and single frequency using the Harmonic Oscillation method. These methods have been verified individually and in combination by comparison with the Harmonic Oscillation method. For small amplitudes of motion, the unsteady flow problem is linear and therefore these methods give accurate results, as expected.

Flutter calculations have been performed for two examples. The first example has only pitching degree of freedom. Calculations have been done over a range of subsonic Mach numbers using a flat plate airfoil and a 5\% thick double-circulararc airfoil. For both airfoils, the flutter reduced frequency is seen to increase with Mach number and the effect of airfoil thickness is to increase the flutter reduced frequency. The results obtained from the present calculations with a flat plate airfoil show some differences when compared with the results from the classical linear theory. These differences are not eliminated by grid refinement indicating that some other cause contributes to this discrepancy. However, there is no indication that the problem lies in the use of either the Influence Coefficient method or Pulse Response method.

The second example for which flutter calculations have been performed is a two-degree-of-freedom system with geometric and structural parameters representative of the SR5 propfan. Once again, calculations have been performed over a subsonic range of Mach numbers. The flutter reduced velocity and the flutter frequency ratio are seen to decrease continuously with increasing Mach number; the decrease in flutter reduced velocity is almost linear with Mach number. The difference between the results for the flat plate and the SR5 airfoil are negligible. It is inferred that the combination of large gap-to-chord ratio, small thickness-to-chord ratio and small camber angle results in very little mean flow deflection and consequently there is very little change in the unsteady aerodynamic behavior due to the addition of loading.

The present approach allows a unified analysis capability in which both time domain and frequency domain flutter calculations can be performed using the same time-marching algorithm. This will allow a direct comparison of results from linear and nonlinear flutter analyses without concern for differences in CFD algorithms, grids and other purely numerical factors. The computational efficiency that is provided by these methods will allow flutter calculations to be done more routinely than was previously possible with the Harmonic Oscillation method. The methods implemented here on a full-potential solver can just as 
easily be applied to other time-marching analyses, such as those based on the Euler equations. Although the present work has been restricted to subsonic Mach numbers, transonic flow calculations can also be performed using the same methods. 


\section{REFERENCES}

1 Mehmed, O., Kaza, K. R. V., Lubomski, J. F., and Kielb, R. E., "BendingTorsion Flutter of a Highly Swept Advanced Turboprop," NASA TM 82975, 1982.

2 Bakhle, M. A., Reddy, T. S. R., Keith, T. G., Jr., "Time Domain Flutter Analysis of Cascades Using a Full-Potential Solver," AIAA Paper 90-0984, Apr. 1990. To be published in AIAA Journal, July 1991.

3 Williams, M. H. and $\mathrm{Ku}, \mathrm{C}$. C., "Three Dimensional Full Potential Aerodynamic Method for the Aeroelastic Modeling of Propfans," AIAA Paper 901120, Apr. 1990.

4 Smith, S. N., "Discrete Frequency Sound Generation in Axial Flow Turbomachines," British Aeronautical Research Council, London, ARC R\&M No. 3709, 1971.

5 Lane, F, "Supersonic Flow Past an Oscillating Cascade with Supersonic Leading-Edge Locus," Journal of the Aeronautical Sciences, Vol. 24, Jan. 1957, pp. 65-66.

6 Whitehead, D. S. and Grant, R. J., "Force and Moment Coefficients for High Deflection Cascades," Proc. 2nd Intl. Symp. on Aeroelasticity in Turbomachines, (ed. P. Suter), Juris-Verlag Zurich, 1981, pp. 85-127.

7 Verdon, J. M. and Caspar, J. R., "Development of a Linear Aerodynamic Analysis for Finite-Deflection Subsonic Cascades," AIAA Journal, Vol. 20, No. 9, Sep. 1982, pp. 1259-1267.

8 Hall, K. C. and Crawley, E. F., "Calculation of Unsteady Flows in Turbomachinery Using the Linearized Euler Equations," AIAA Journal, Vol. 27, No. 6, June 1989, pp. 777-787.

9 Kao, Y. F., "A Two-Dimensional Unsteady Analysis for Transonic and Supersonic Cascade Flows," Ph.D. Thesis, School of Aeronautics and Astronautics, Purdue University, West Lafayette, Indiana, May 1989.

10 Huff, D. L., "Numerical Analysis of Flow Through Oscillating Cascade Sections," AIAA Paper 89-0437, Jan. 1989.

11 Reddy, T. S. R., Bakhle, M. A., and Huff, D. L., "Flutter Analysis of a Supersonic Cascade in Time Domain Using an Euler Solver," NASA TM in preparation, 1990.

12 Bakhle, M. A., Keith, T. G. Jr., and Kaza, K. R. V., "Application of a FullPotential Solver to Bending-Torsion Flutter in Cascades," AIAA Paper 89-1386, Apr. 1989. 
13 Reddy, T. S. R., Srivastava, R., and Kaza, K. R. V., "The Effects of Rotational Flow, Viscosity, Thickness, and Shape on Transonic Flutter Dip Phenomena," AIAA Paper 88-2348, Apr. 1988.

14 Bisplinghoff, R. L. and Ashley, H., "Principles of Aeroelasticity," John Wiley and Sons, Inc., New York, 1962.

15 Shankar, V., Ide, H., Gorski, J., and Osher, S., "A Fast Time-Accurate Unsteady Full Potential Scheme," AIAẢ Paper 85-1512, Aug. 1985.

16 Hedstrom, G. W., "Nonreflecting Boundary Conditions for Nonlinear Hyperbolic Systems," Journal of Computational Physics, Vol. 30, 1979, pp. 222-237.

17 Lane, F., "System Mode Shapes in the Flutter of Compressor Blade Rows," Journal of the Aeronautical Sciences, Vol. 23, Jan. 1956, pp. 54-66.

18 Bendiksen, O., and Friedmann, P., "Coupled Bending-Torsion Flutter in Cascades," AIAA Journal, Vol. 18, No. 2, Feb. 1980, pp. 194-201.

19 Nixon, D., Tzuoo, K. L., and Ayoub, A., "Rapid Computation of Unsteady Transonic Cascade Flows," AIAA Journal, Vol. 25, No. 5, May 1987, pp. 760-762.

20 Buffum, D. H., and Fleeter, S., "Aerodynamics of a Linear Oscillating Cascade," NASA TM 103250, Aug. 1990.

21 Ballhaus, W. F. and Goorjian, P. M., "Computation of Unsteady Transonic Flows by the Indicial Method," AIAA Journal, Vol. 16, No. 2, Feb. 1978, pp. 117 . 124.

22 Kerlick, G. D. and Nixon, D., "A High-Frequency Transonic Small Disturbance Code for Unsteady Flows in a Cascade," AlAA Paper 82-0955, June 1982.

23 Myers, M. R. and Ruo, S. Y., "Calculation of Unsteady Aerodynamic Coefficients Using Transonic Time Domain Methods," AIAA Paper 83-0885, Apr. 1983.

24 Davies, D. E. and Salmond, D. J., "Indicial Approach to Harmonic Perturbations in Transonic Flow," AIAA Journal, Vol. 18, No. 8, Aug. 1980, pp. 1012-1014.

25 Seidel, D. A., Bennett, R. M., and Whitlow, W., Jr., "An Exploratory Study of Finite-Difference Grids for Transonic Unsteady Aerodynamics," AIAA Paper 83 0503, Jan. 1983. 


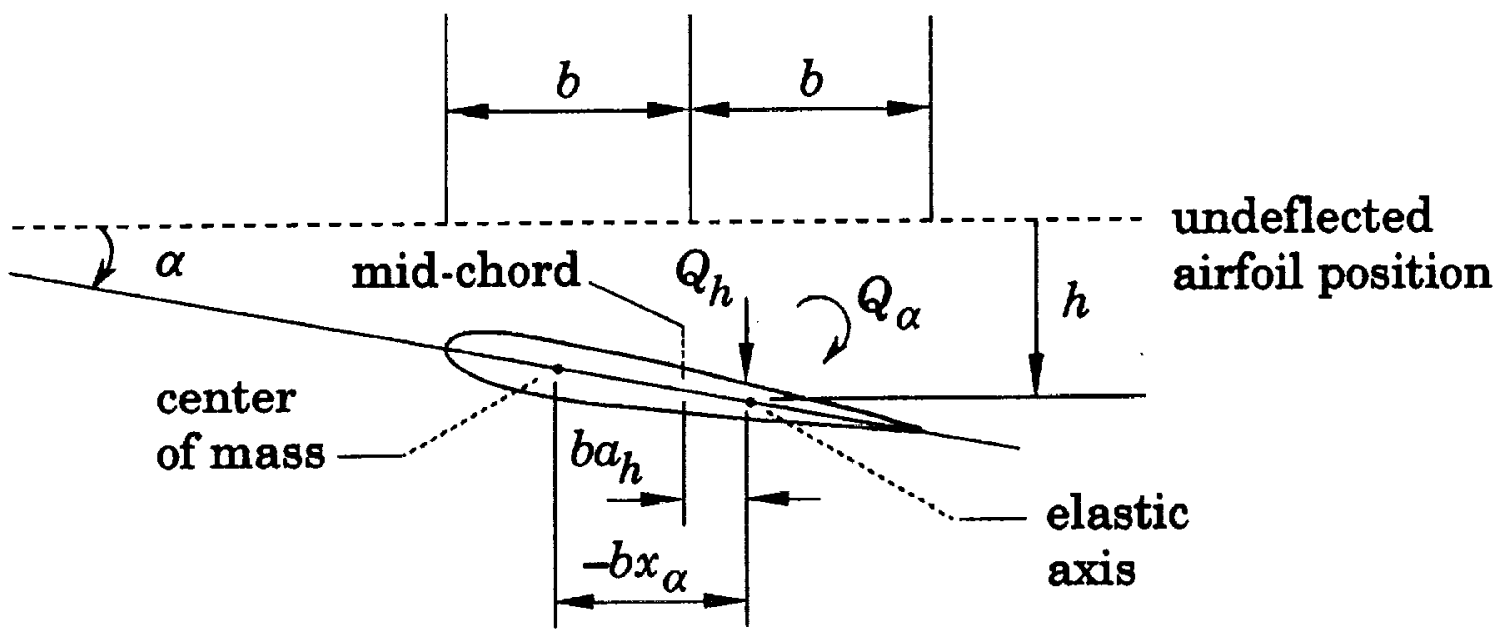

Figure 1a: Typical section blade model with two degrees-of-freedom.

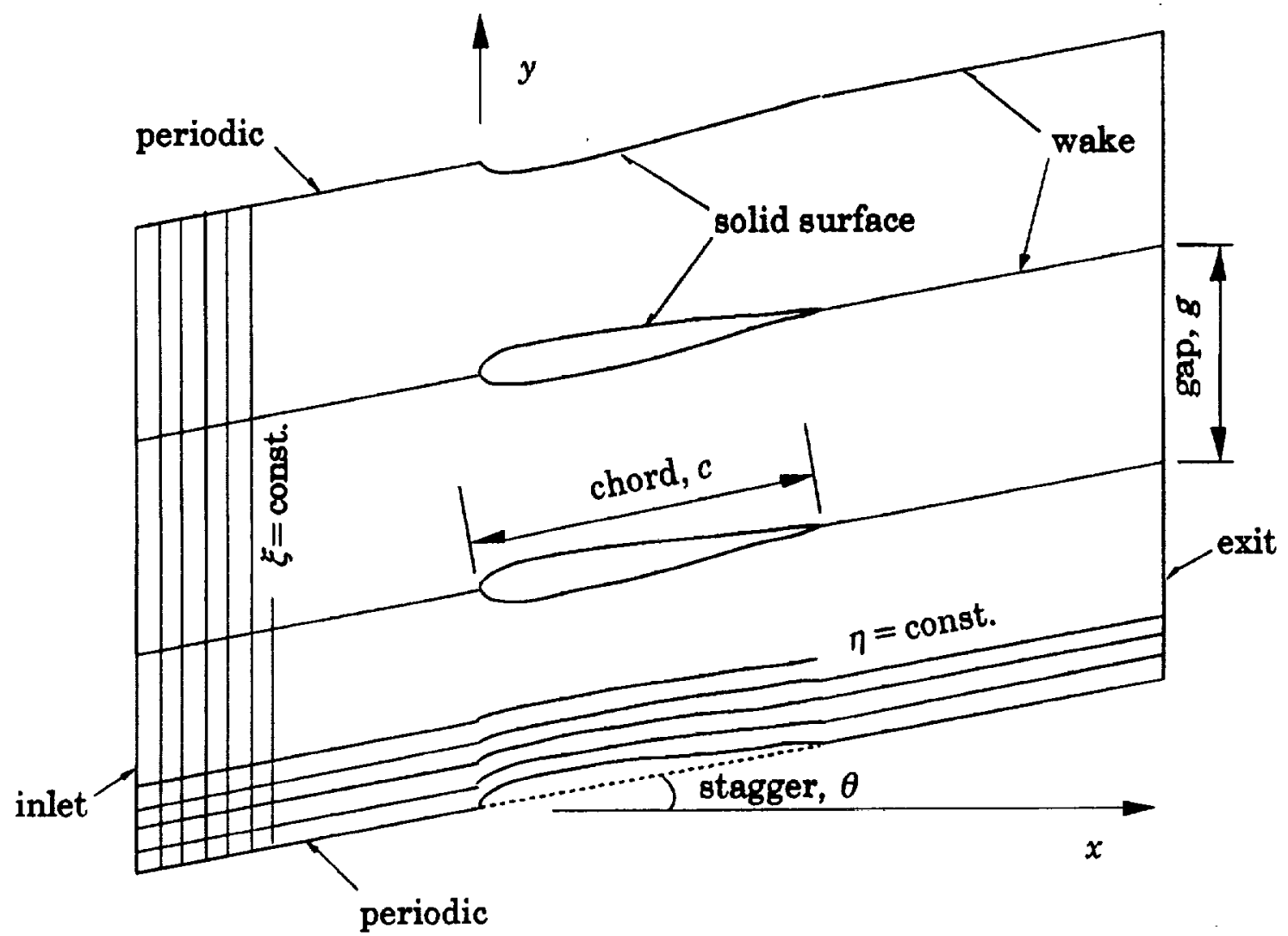

Figure 1b: Cascade geometry and schematic of H-type grid. 


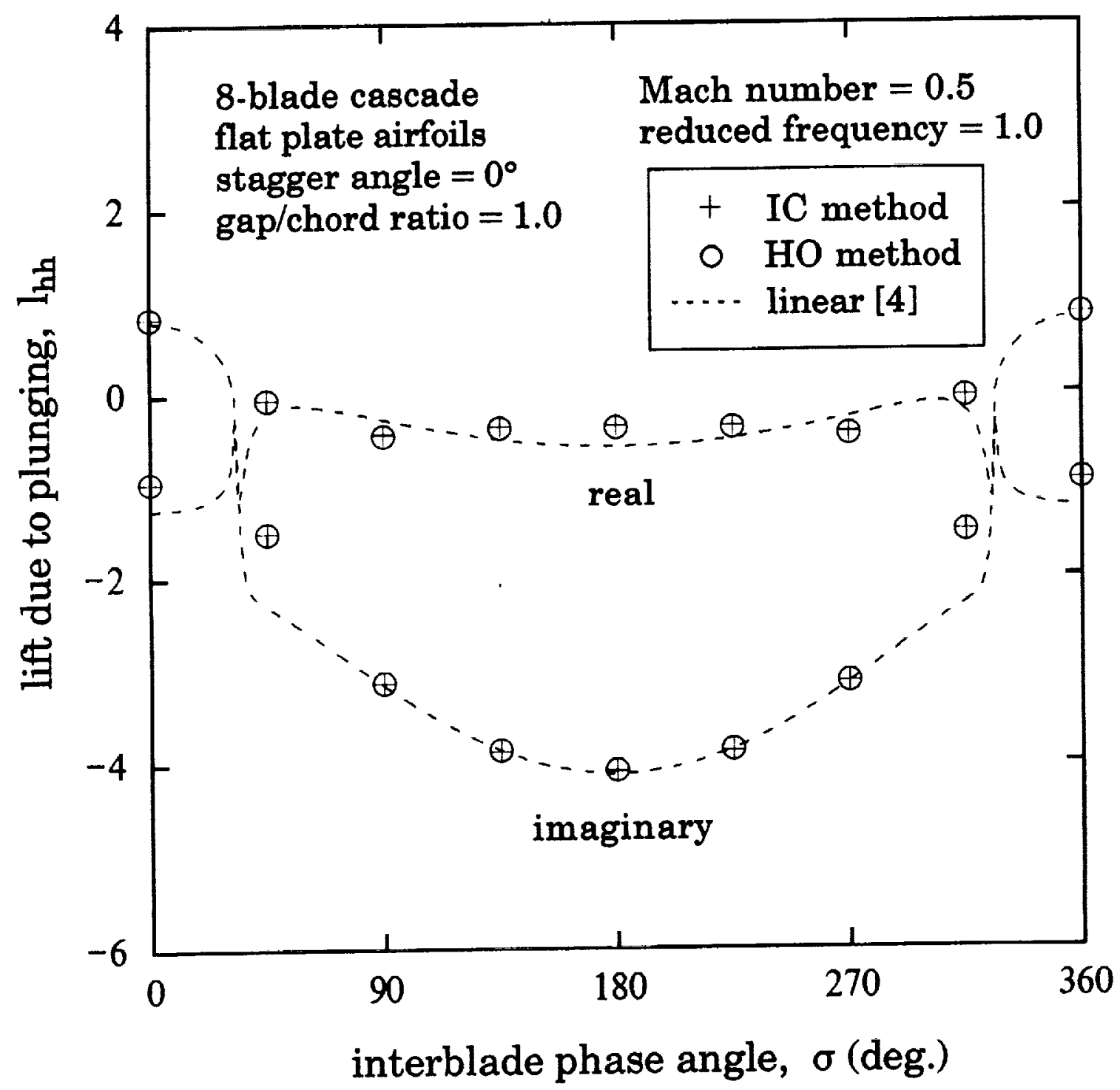

Figure 2: Variation of lift due to plunging with phase angle. 


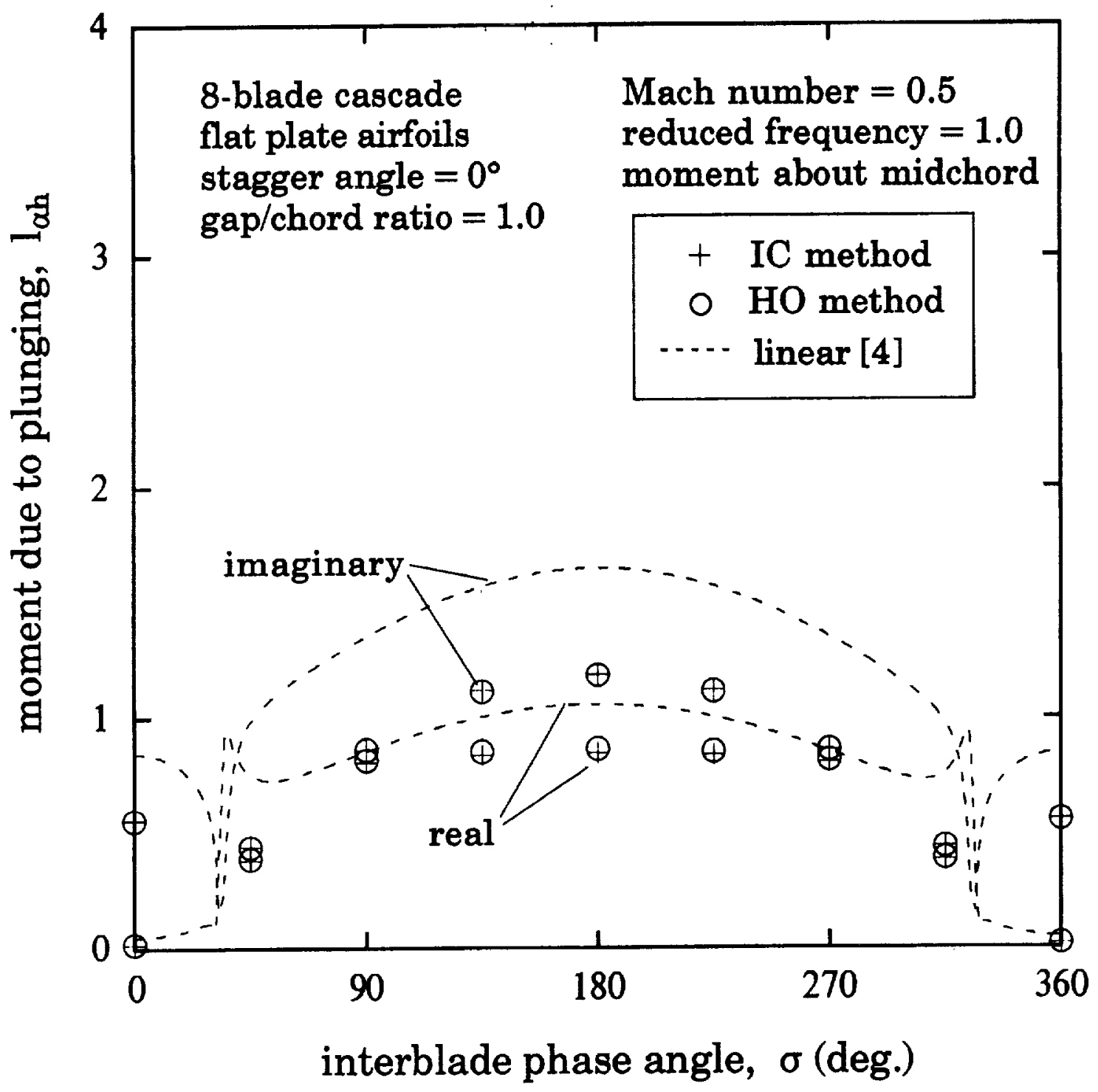

Figure 3: Variation of moment due to plunging with phase angle. 


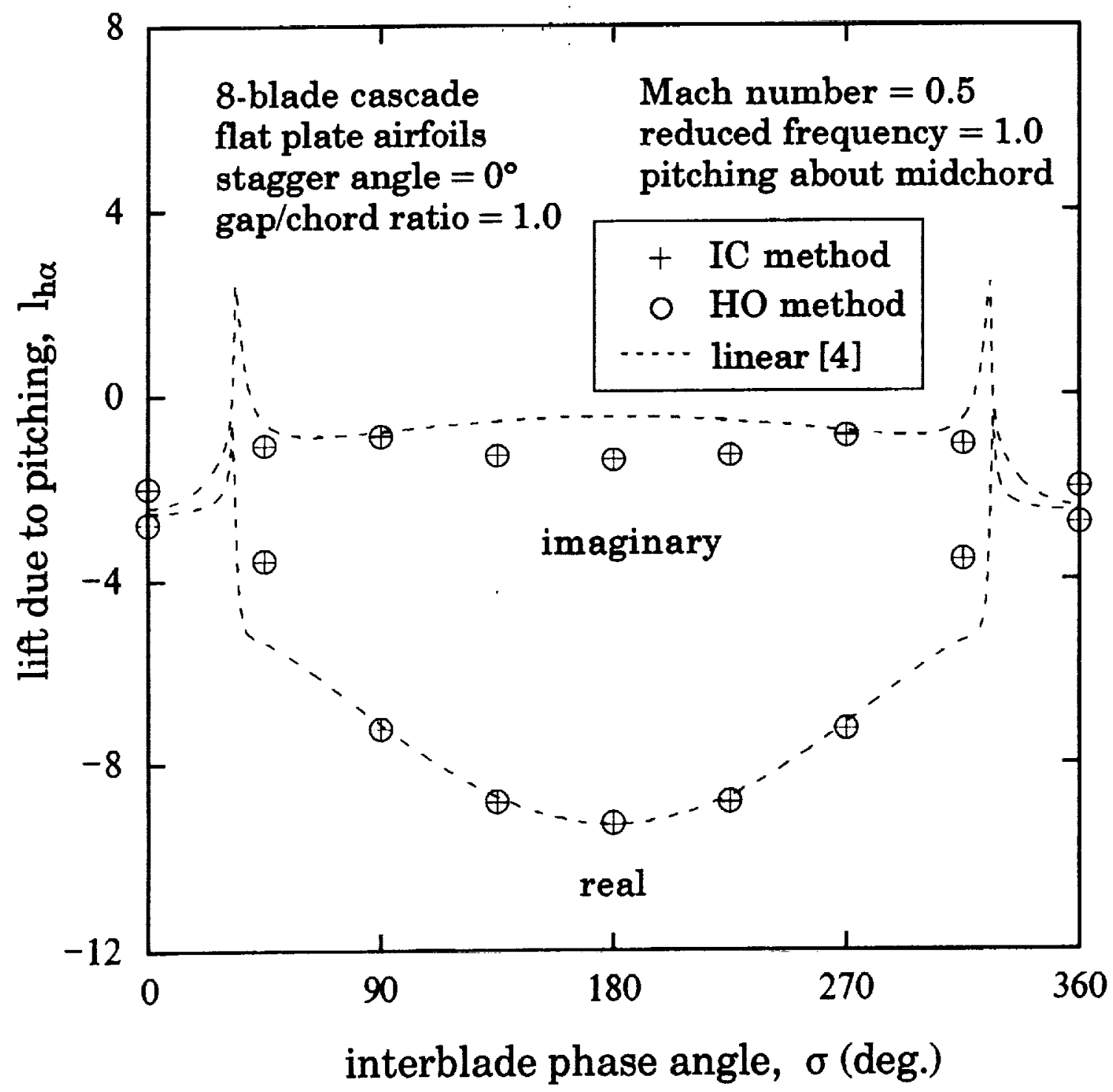

Figure 4: Variation of lift due to pitching with phase angle. 


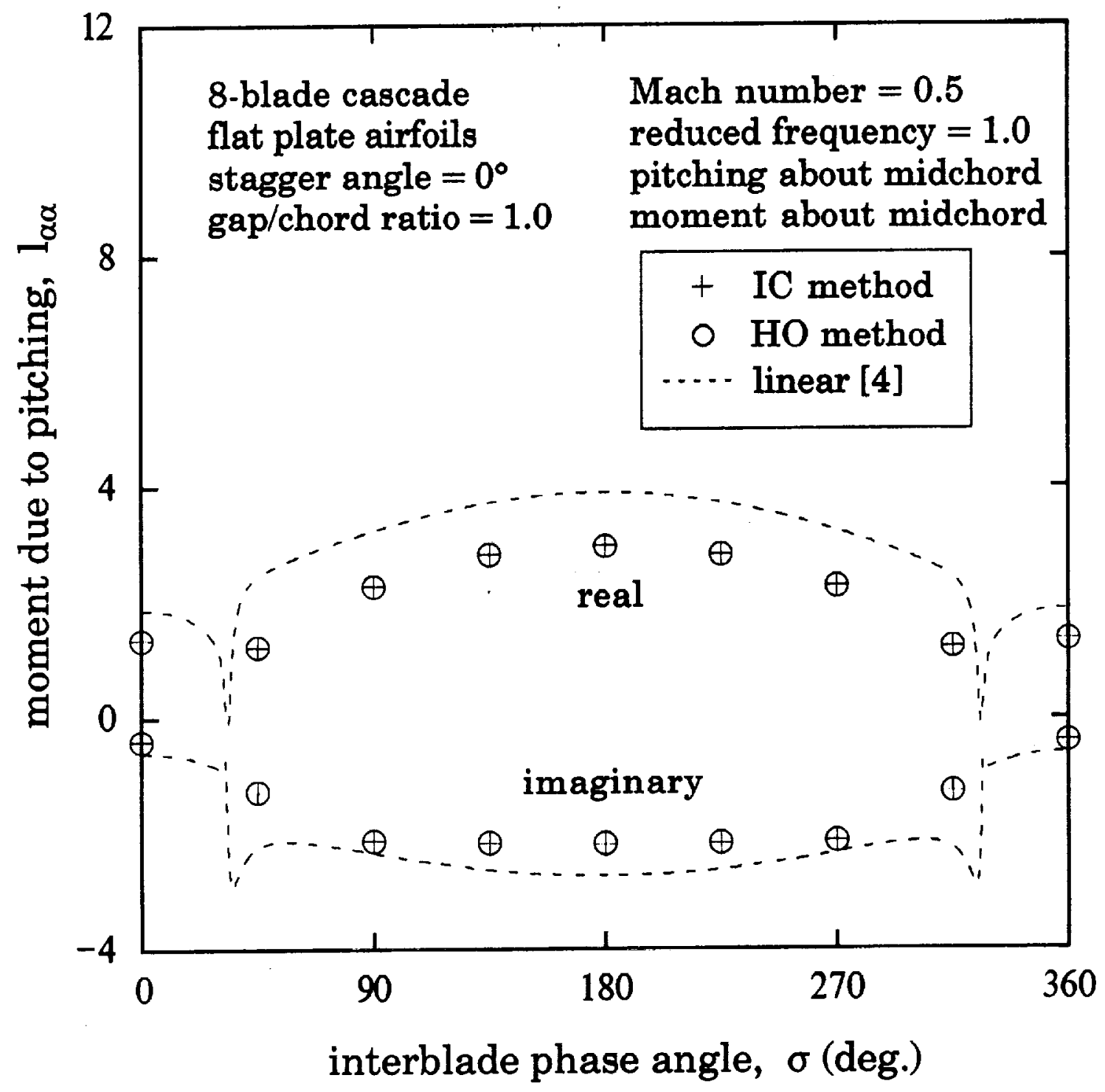

Figure 5: Variation of moment due to pitching with phase angle. 

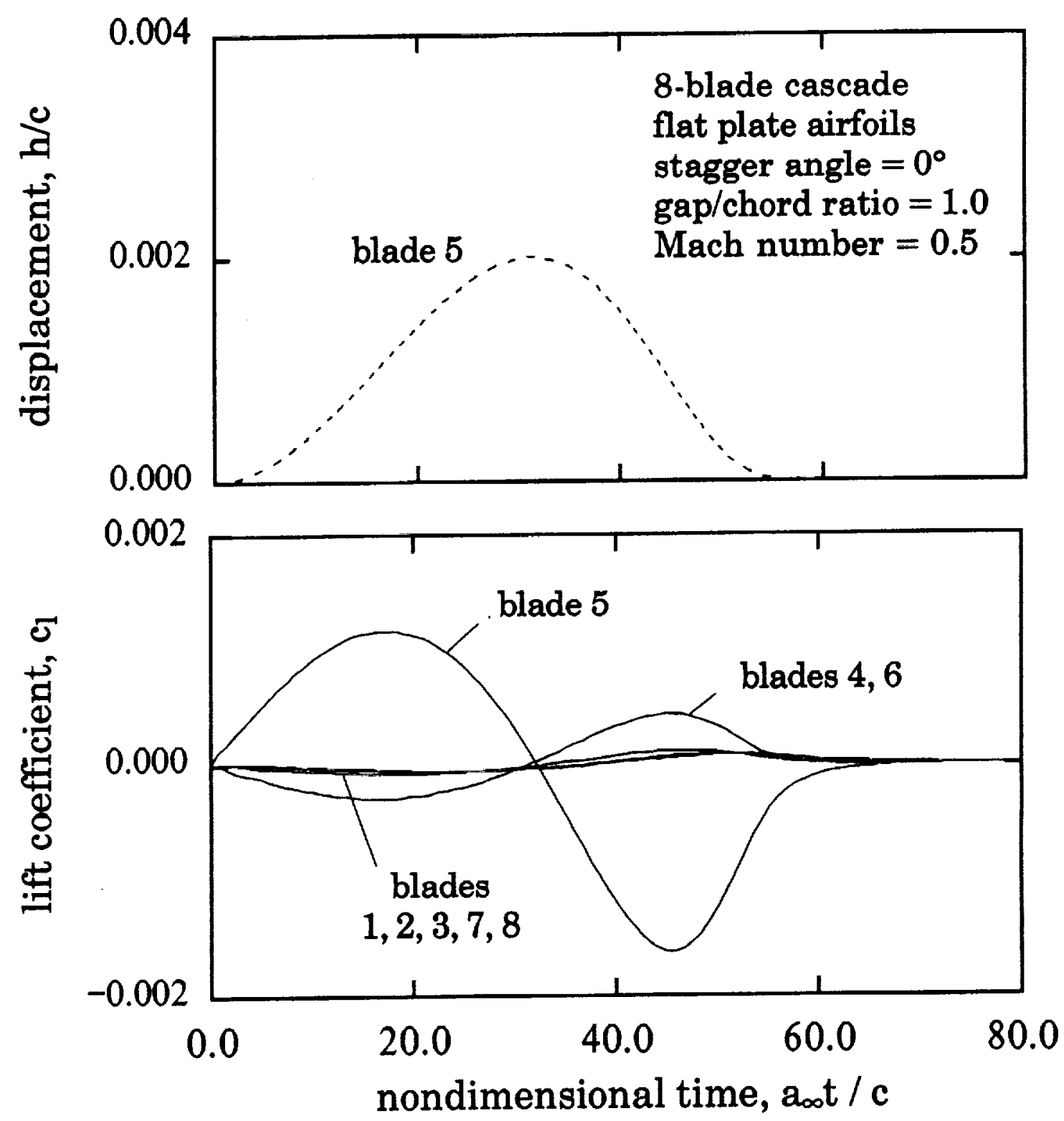

Figure 6: Time history of displacement and lift in Pulse Response method. 


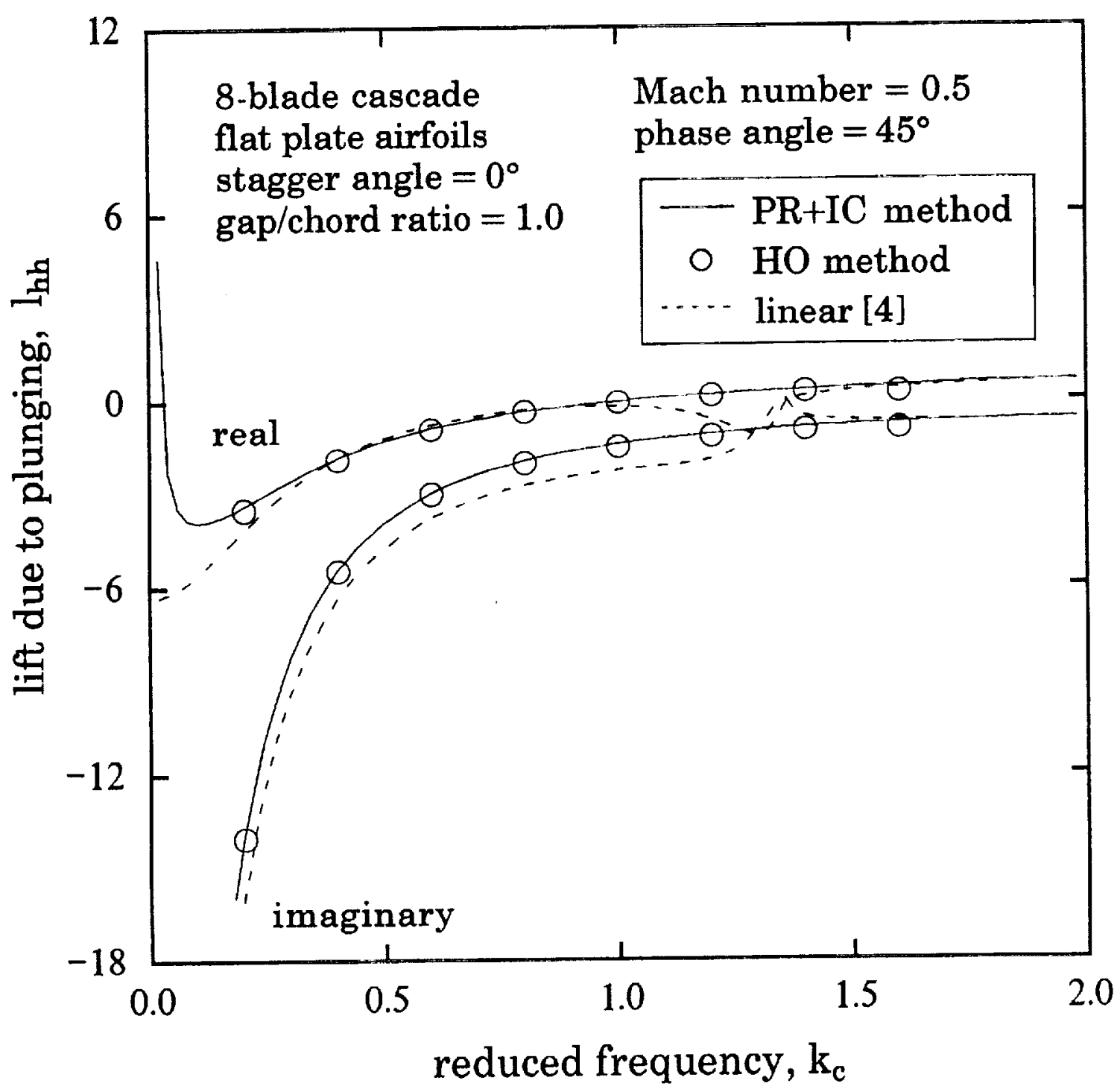

Figure 7: Variation of lift due to plunging with reduced frequency. 


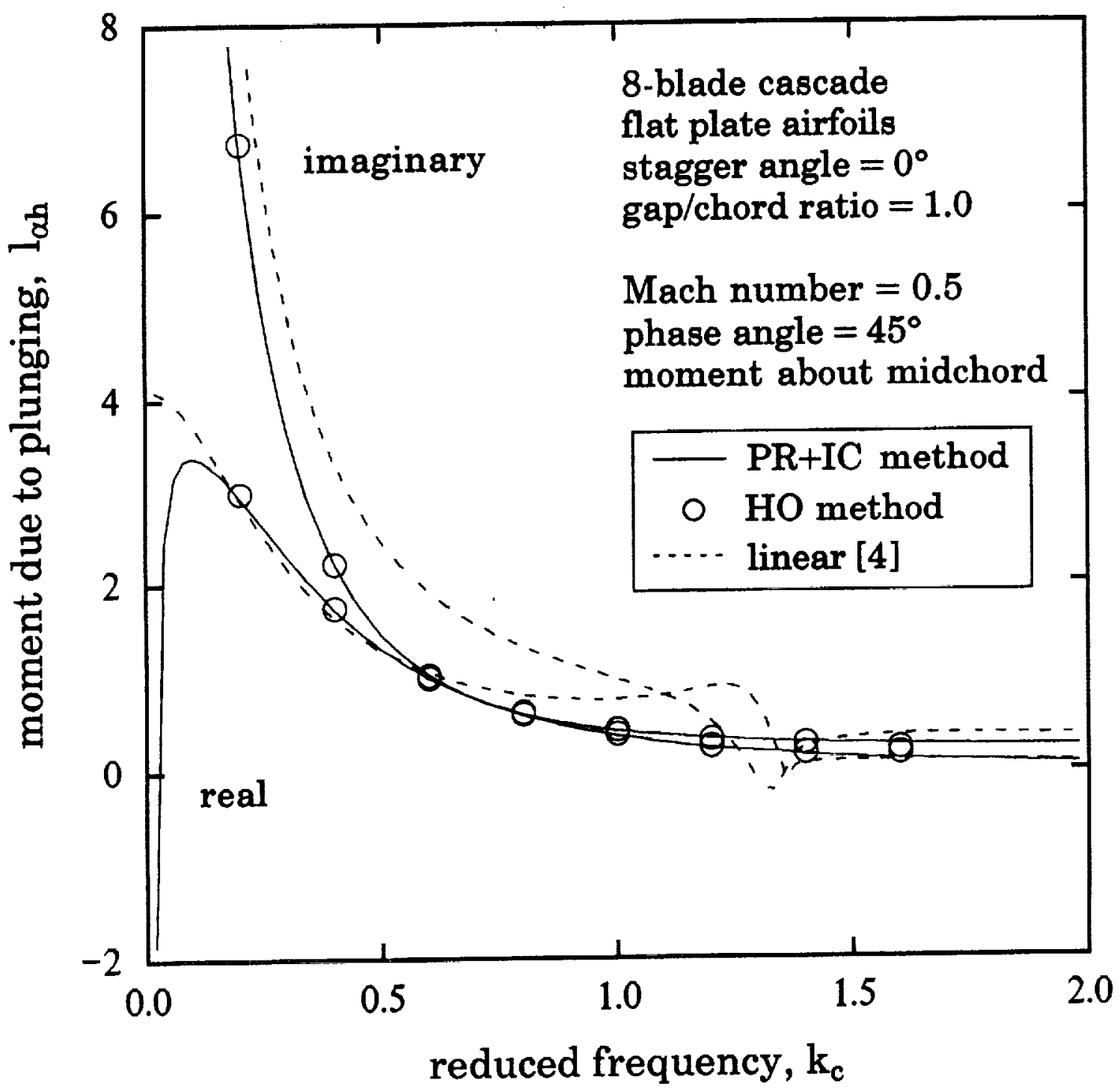

Figure 8: Variation of moment due to plunging with reduced frequency. 


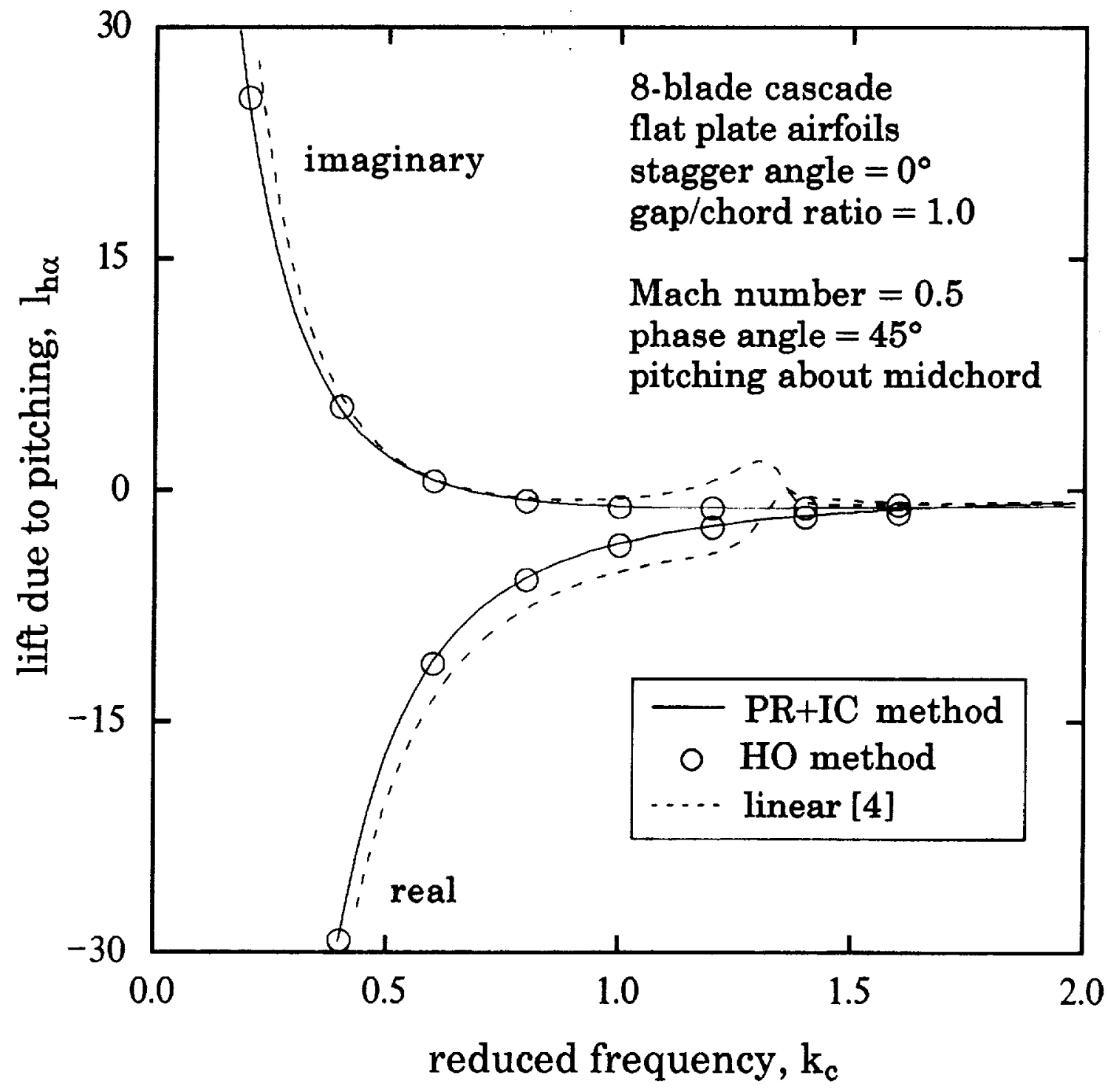

Figure 9: Variation of lift due to pitching with reduced frequency. 


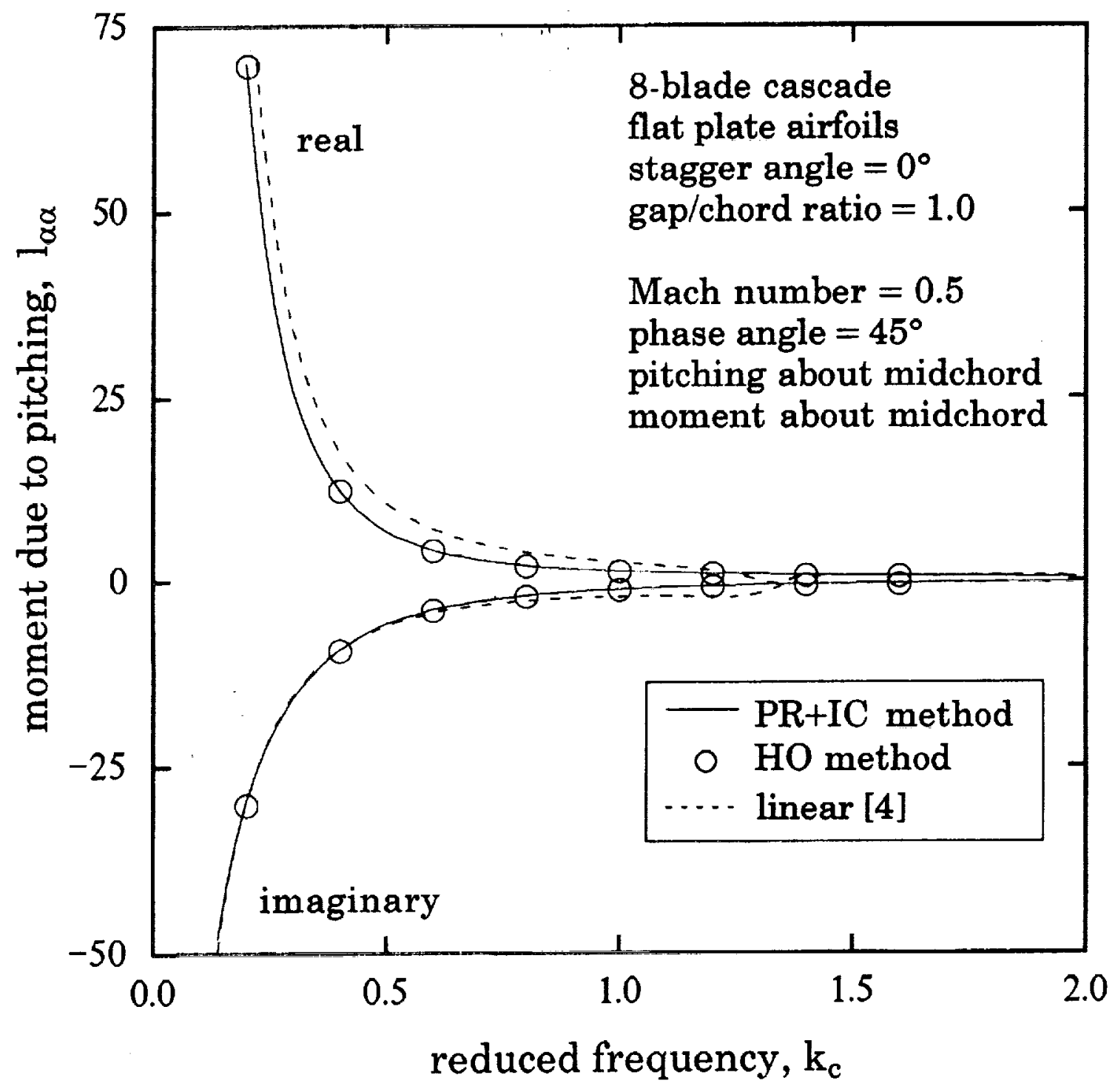

Figure 10: Variation of moment due to pitching with reduced frequency. 


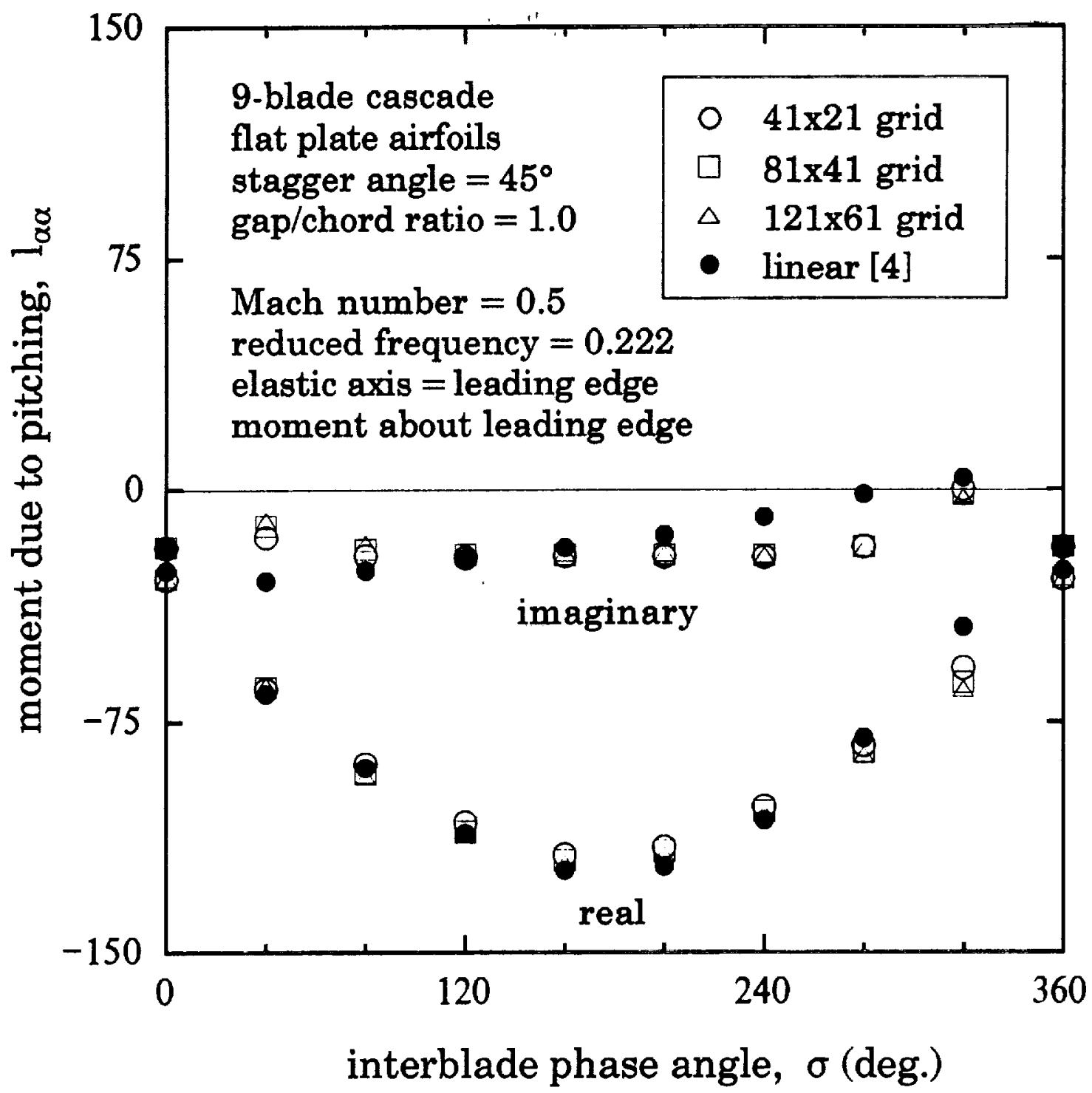

Figure 11: Variation of moment due to pitching with interblade phase angle, example 1. 


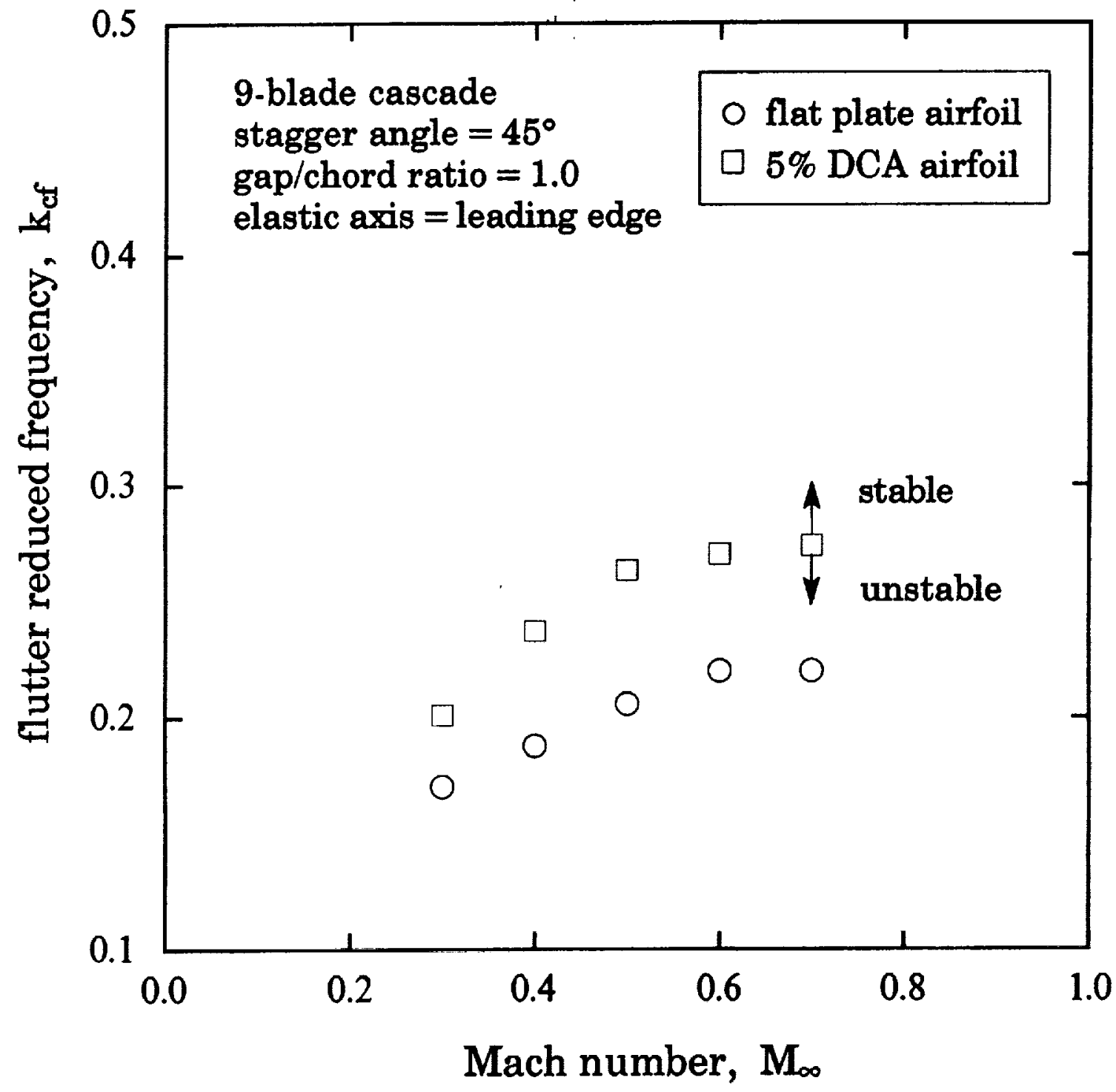

Figure 12: Variation of flutter reduced frequency with Mach number, example 1. 


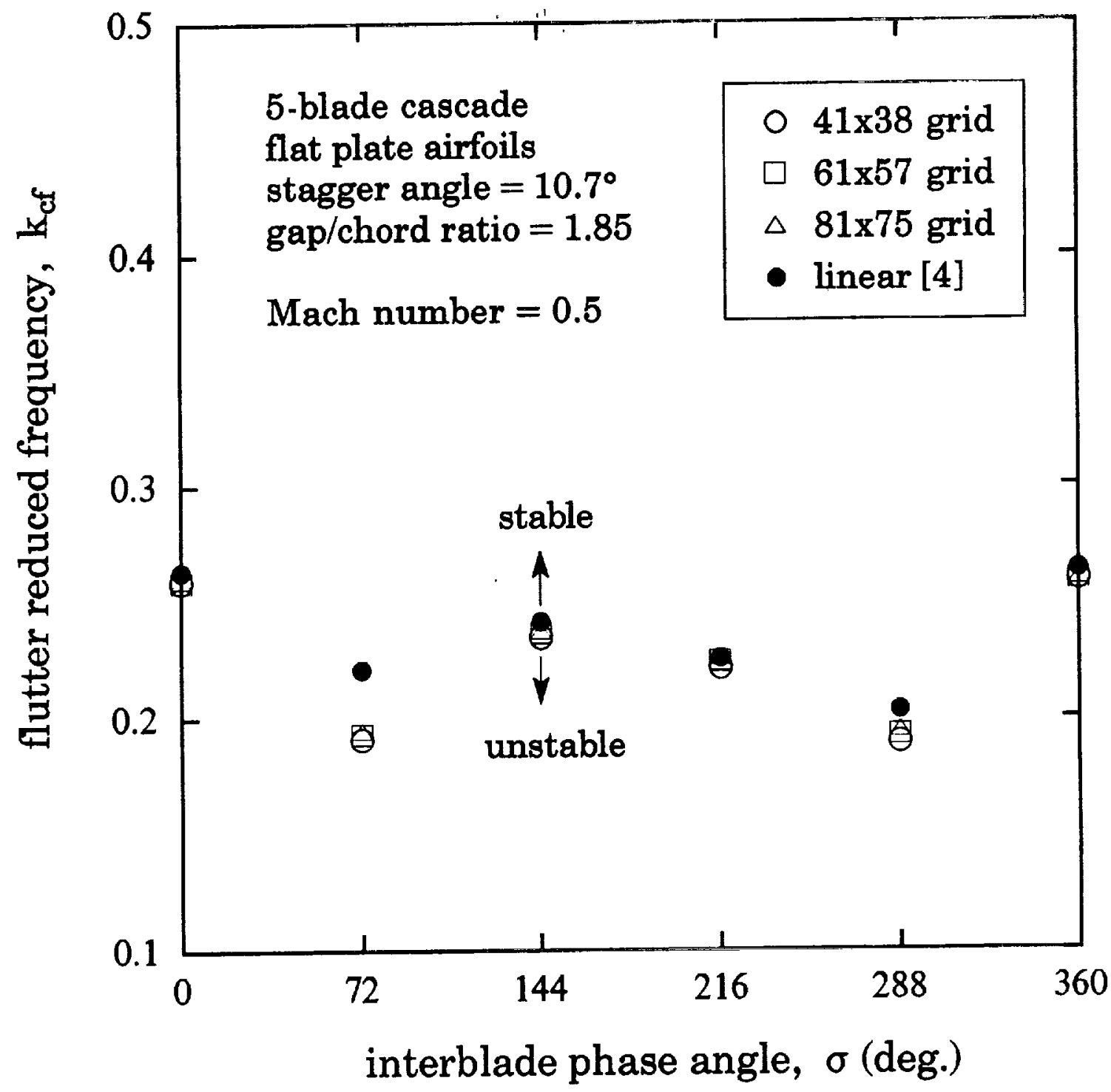

Figure 13: Variation of flutter reduced frequency with interblade phase angle, example 2. 


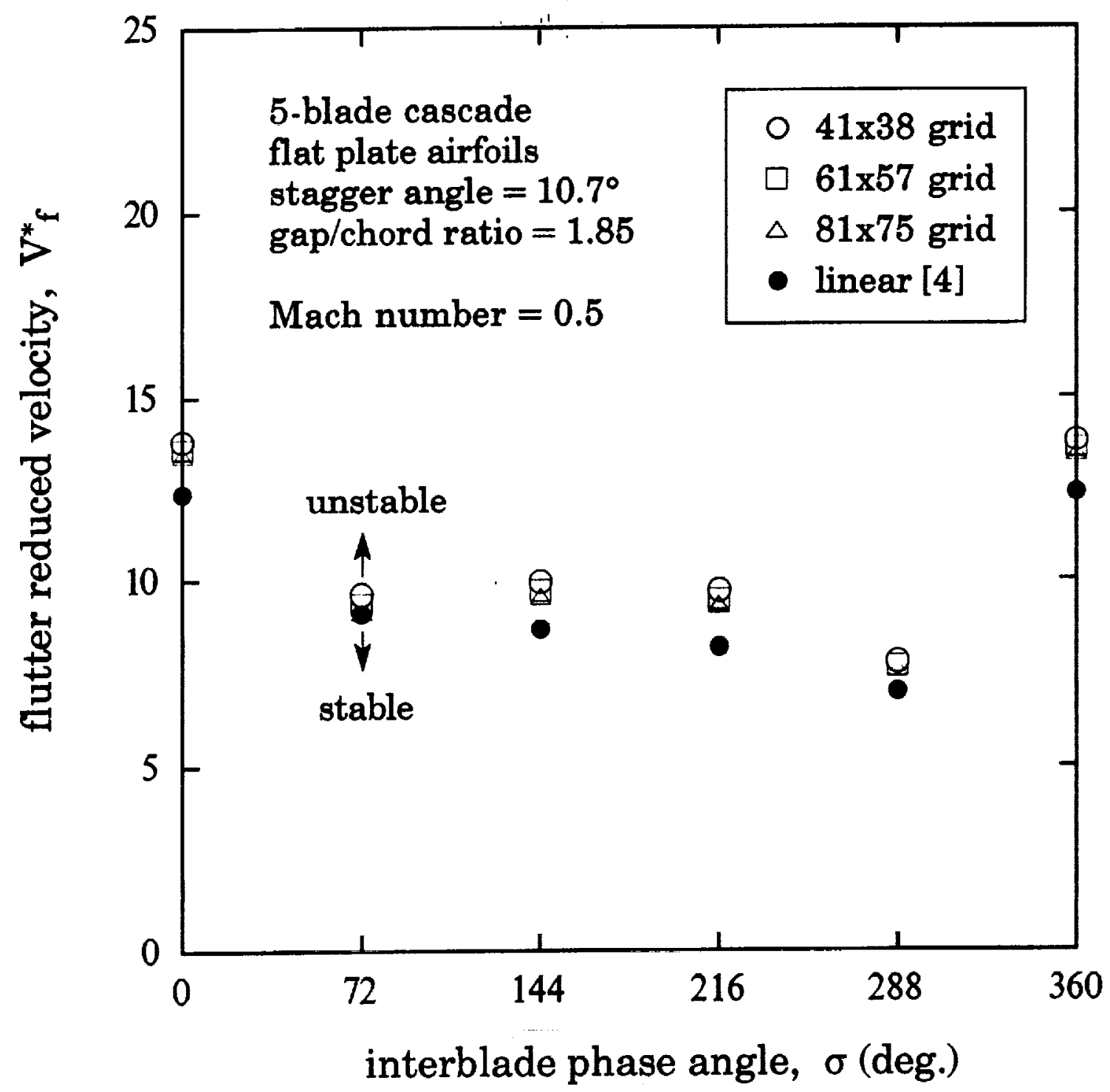

Figure 14: Variation of flutter reduced velocity with interblade phase angle, example 2. 


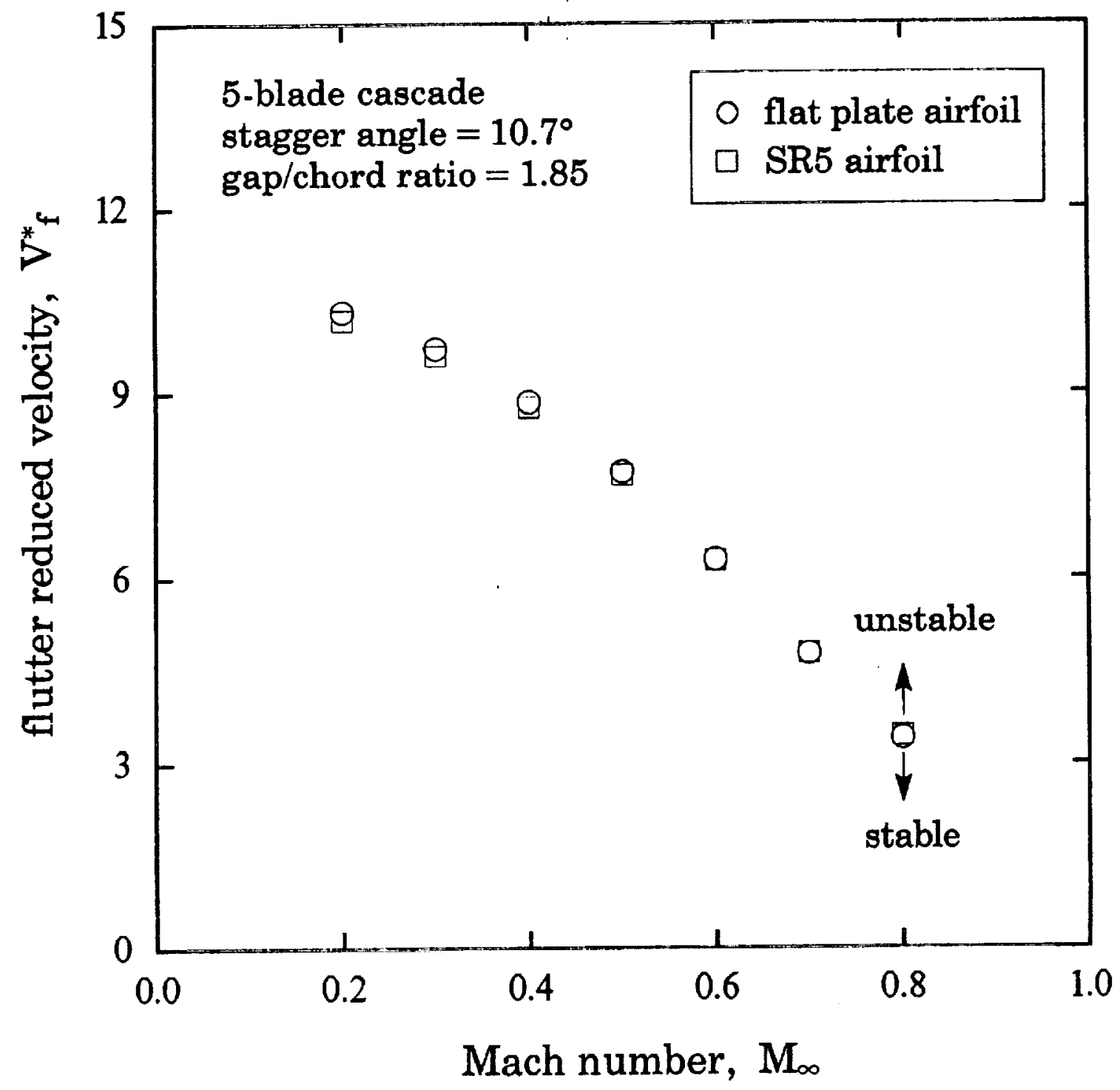

Figure 15: Variation of flutter reduced velocity with Mach number, example 2. 


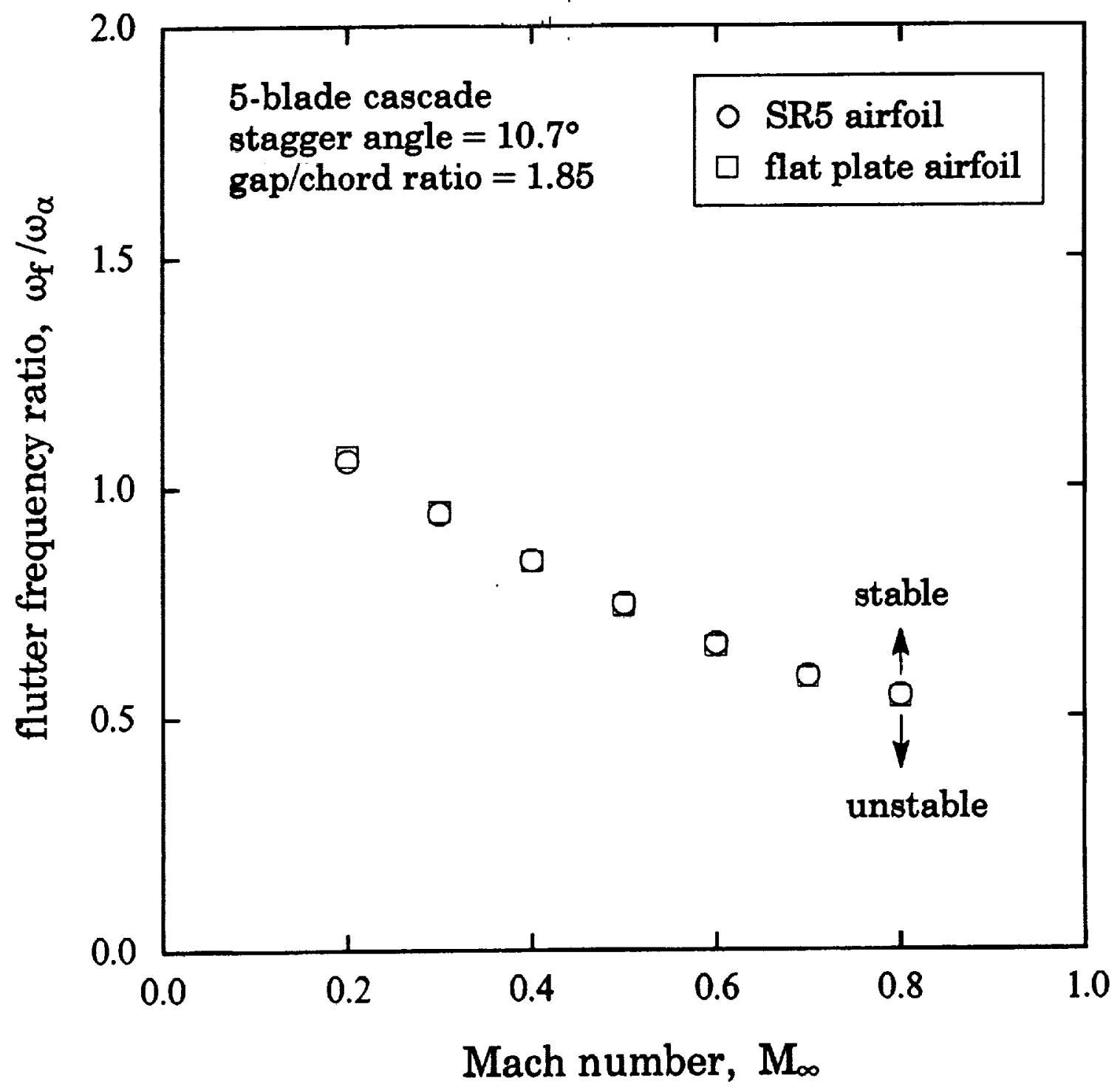

Figure 16: Variation of flutter frequency ratio with Mach number, example 2. 



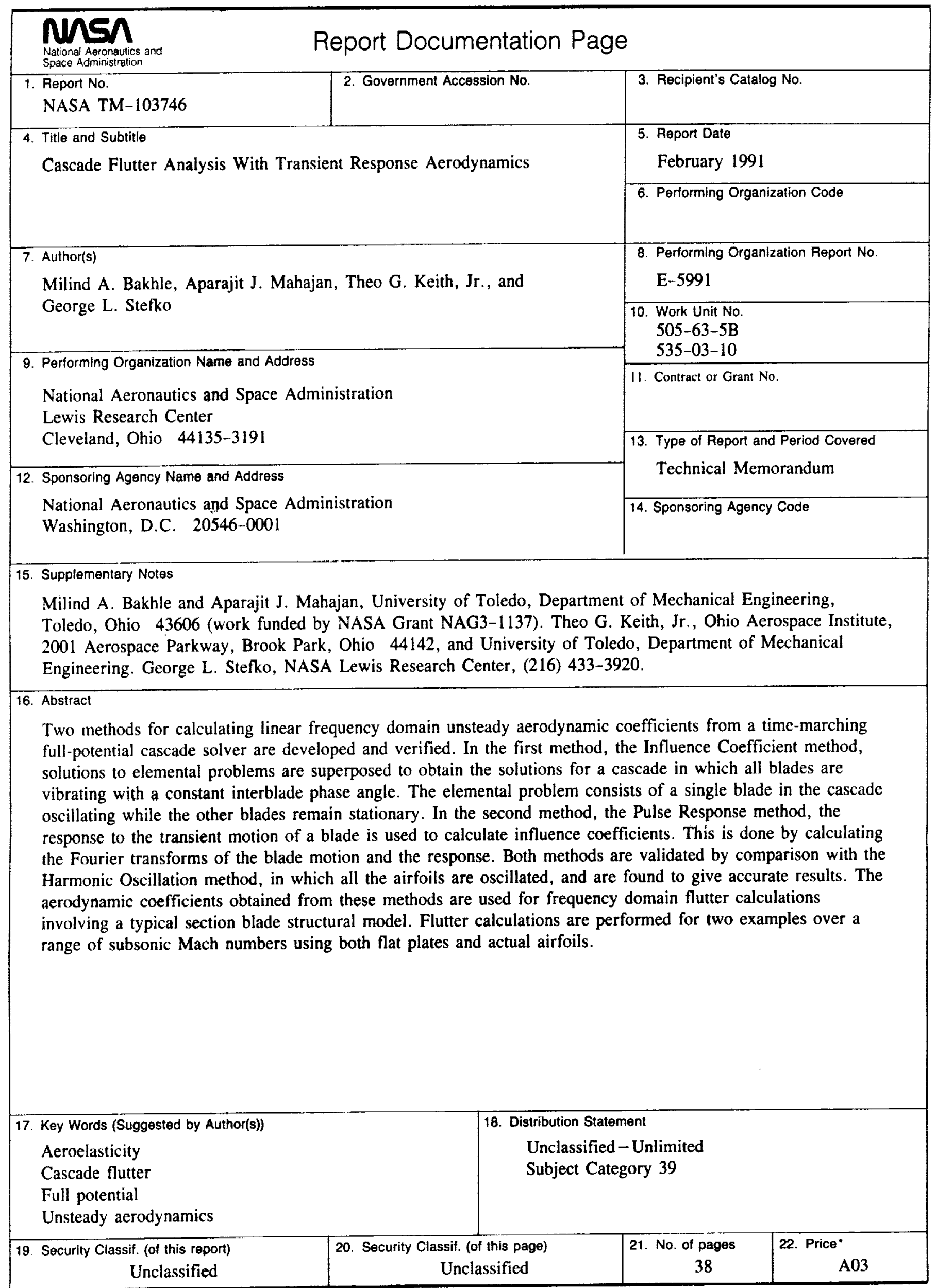

